The Journal of Symbolic Logic

Volume 86, Number 4, December 2021

\title{
HIGHER MILLER FORCING MAY COLLAPSE CARDINALS
}

\author{
HEIKE MILDENBERGER AND SAHARON SHELAH (D)
}

\begin{abstract}
We show that it is independent whether club $\kappa$-Miller forcing preserves $\kappa^{++}$. We show that under $\kappa^{<\kappa}>\kappa$, club $\kappa$-Miller forcing collapses $\kappa^{<\kappa}$ to $\kappa$. Answering a question by Brendle, Brooke-Taylor, Friedman and Montoya, we show that the iteration of ultrafilter $\kappa$-Miller forcing does not have the Laver property.
\end{abstract}

§1. Introduction. Many of the tree forcings on the Baire space over $\omega$ have various analogues for higher cardinals. Here we are concerned with club $\kappa$-Miller forcing [16] for regular uncountable $\kappa$, and some of our results apply also to $\kappa$-Sacks forcing and some higher amoeba forcings.

In Section 2 we review some of the common tree higher forcings for uncountable $\kappa$. We show that the diamond principle and the inaccessibility of $\kappa$ can be replaced by the weaker principle $(D l)_{\kappa}$ in the known theorems about the preservation of $\kappa^{+}$in $\kappa$-supported iterations of some popular higher tree forcings like Kanamori's $\kappa$-Sacks forcing [10], Landver's versions of filter Sacks forcing [14], $\kappa$-club Miller forcing [7], and $\kappa$-club Laver forcing [4].

In Section 3, we show that under $\kappa^{<\kappa}=\kappa$ it is independent whether club $\kappa$-Miller forcing preserves $2^{\kappa}=\kappa^{++}$. Petr Simon proved that Sacks forcing collapses the continuum to the bounding number. Under an hypothesis on a $\kappa$-evasion number, we show that club Miller forcing collapses $2^{\kappa}$ to the bounding number at $\kappa$.

In Section 4 we prove that club $\kappa$-Miller collapses $\kappa^{<\kappa}$ to $\kappa$ if $\kappa^{<\kappa}>\kappa$. This may serve as a mathematical reason for the assumption $\kappa^{<\kappa}=\kappa$ in work with higher tree forcings.

In Section 5 we answer a question of Brendle, Brooke-Taylor, Friedman and Montoya: We show that the $(\leq \kappa)$-support iteration of $\kappa$-Miller forcing with branching into a normal ultrafilter does not have the Laver property.

Notation: Throughout the paper we let $\kappa$ be an uncountable regular cardinal. When used, we add additional conditions on $\kappa$. If $\operatorname{dom}(t), i$ are ordinals, we write $t^{\wedge}\langle i\rangle$ for the concatenation of $t$ with the singleton function $\{(0, i)\}$, i.e., $t^{\wedge}\langle i\rangle=$ $t \cup\{(\operatorname{dom}(t), i)\}$. We write ${ }^{\lambda>} \kappa$ for the set of functions $f: \alpha \rightarrow \kappa$ for some $\alpha<\lambda$. The domain $\alpha$ of $f$ is also called the length of $f$. The set of subsets of $\kappa$ of size $\kappa$ is denoted by $[\kappa]^{\kappa}$.

Received April 24, 2020.

2020 Mathematics Subject Classification. 03E05, 03E04, 03E15.

Key words and phrases. forcing with higher perfect trees.

(C) The Author(s), 2021. Published by Cambridge University Press on behalf of Association for Symbolic Logic. This is an Open Access article, distributed under the terms of the Creative Commons Attribution licence (https://creativecommons.org/licenses/by/4.0/), which permits unrestricted re-use, distribution, and reproduction in any medium, provided the original work is properly cited. 
DEFINITION 1.1. Let $\kappa$ be an infinite cardinal.

(1) A tree $($ on $\kappa)$ is a non-empty subset of ${ }^{\kappa>} \kappa$ that is closed under initial segments. We use the symbol $\unlhd$ for the initial segment relation and the symbot $\triangleleft$ for the corresponding strict relation.

(2) A tree on $\kappa$ is called unbounded if

$$
(\forall t \in p)(\forall \alpha<\kappa)\left(\exists t^{\prime} \in p\right)\left(\operatorname{dom}\left(t^{\prime}\right) \geq \alpha \wedge t^{\prime} \unrhd t\right) .
$$

(3) Let $T \subseteq{ }^{\kappa>} \kappa$ be a tree and $s \in T$. We let

$$
T^{\langle s\rangle}=\{t \in T: t \unlhd s \vee s \unlhd t\} .
$$

(4) We let $[T]=\left\{x \in{ }^{\kappa} \kappa:(\forall \alpha<\kappa)(x \uparrow \alpha \in T)\right\}$. The set $[T]$ is called the set of branches of $T$ or the body of $T$.

(5) The elements of a tree are called nodes. A node that has at least two immediate $\triangleleft$-successors in $p$ is called a splitting node of $p$. The set of splitting nodes of $p$ is denoted by $\operatorname{spl}(p)$.

(6) Let $T \subseteq{ }^{\kappa>} \kappa$ be a tree that contains a splitting node. We let the trunk of $T$, $\operatorname{tr}(T)$, be the $\unlhd$-least splitting node of $T$. If $T$ has no splitting node then we let $\operatorname{tr}(p)=p$.

(7) Let $\mathbb{P}=\left(\mathbb{P}, \leq_{\mathbb{P}}\right)=(\mathbb{P}, \supseteq)$ denote a forcing whose conditions are $(<\kappa)$-closed unbounded trees. Let $G$ be a $P$-generic filter. In $\mathbf{V}[G]$ we define

$$
x_{G}=\bigcup\{\operatorname{tr}(p): p \in G\} .
$$

The function $x_{G}$ is called the generic $\kappa$-real, branch, or function.

In the forcings we investigate, the conditions are unbounded trees with additional properties. Subtrees are stronger conditions. In forcing we use the Israeli notation that $p \leq_{\mathbb{P}} q$ means that $q$ is stronger than $p .{ }^{1}$ We usually write $q \subseteq p$. We write $\mathbb{P} \Vdash \varphi$ if any condition (equivalently, the weakest condition is there is such one) forces $\varphi$.

We review some general properties. Often the $\kappa$-real $x_{G}$ is closely related to the generic filter $G$ :

Proposition 1.2. Let $\mathbb{P}=(\mathbb{P}, \supseteq)$ be a forcing whose conditions are $(<\kappa)$-closed unbounded trees $p \subseteq{ }^{\kappa>} \kappa$. In addition we assume that $\mathbb{P}$ contains for each $p \in \mathbb{P}$ and $s \in p$ also the condition $p^{\langle s\rangle}$ (from Definition 1.1(3)). Let $G$ be the generic filter and $x_{G}$ be the generic real. Then the following holds.

(1) For any $p, q \in \mathbb{P}, p \Vdash x_{G} \in[q]$ implies $p \subseteq q$.

(2) For any $p, q \in \mathbb{P}, x_{G} \in[p] \cap[q]$ implies that $p$ and $q$ are compatible.

(3) The $\kappa$-real $x_{G}$ determines $G$ via $G=\left\{p \in \mathbb{P}: x_{G} \in[p]\right\}$.

Proof. (1) If $p \nsubseteq q$ then there is $t \in p \backslash q$, and $p^{\langle t\rangle} \Vdash x_{G} \notin[q]$.

(2) Let $\left.D_{p, q}=\left\{r \in \mathbb{P}: r \Vdash x_{G} \in[p] \cap[q] \vee r \Vdash x_{G} \notin \tilde{[} p\right] \vee r \Vdash x_{G} \notin[q]\right\}$. The set $D_{p, q}$ is dense in $\mathbb{P}$. So there is $r \in D_{p, q} \cap G$. We fix such an $r$. Since $\mathbb{P}$ contains only $(<\kappa)$-closed unbounded trees, we have $x_{G} \in[p] \cap[q] \cap{ }^{\kappa} \kappa$. By the definition of $D_{p, q}, r \Vdash x_{G} \in[p] \cap[q]$. Hence by $(1), r \geq p, q$.

\footnotetext{
${ }^{1}$ We will also observe the alphabetical convention: Letters later in the alphabet are used for stronger conditions.
} 
(3) Suppose $p \in G$. Then by definition $x_{G} \in[p]$. By (2), the set $\left\{p: x_{G} \in[p]\right\}$ is a filter. Since the generic $G$ is a subset of this filter, the latter coincides with $G$. $\dashv$

\$2. On the club version $\kappa$-Miller forcing. For an uncountable cardinal $\kappa$ such that $\kappa^{<\kappa}=\kappa$ and such that $\nabla_{\kappa}$ holds, Kanamori [10] defined a club version of $\kappa$ Sacks forcing, proved a $\kappa$-version of Axiom A for it and iterated it with support of size $\kappa$, in the style of Baumgartner and Laver [3]. Later Eisworth [6] (again under $\kappa^{<\kappa}=\kappa>\omega$ and $\nabla_{\kappa}$ ) and Rosłanowski and Shelah [17-20] (mainly for inaccessible $\kappa)$ investigated versions of $\kappa$-properness and $\left(<\kappa^{+}\right)$-support iterations of iterands of length $\kappa^{++}$for iterands that are in a strong sense proper for the higher cardinal. All of the settings suppose that the iterands are $(<\kappa)$-closed. An early use of $\nabla_{\kappa}$ for showing that the $\kappa$-supported product of $\kappa$-Silver forcing preserves $\kappa^{+}$is in Baumgartners proof of [2, Theorem 6.7]. ${ }^{2}$

A careful inspection shows that in the named examples the diamond principle can be replaced by the weaker principle $(D l)_{\kappa}$ (see Def. 2.8). We mention here some results that show that $(D l)_{\kappa}$ is "slightly weaker": For $\kappa \geq \aleph_{1}$ being a successor cardinal, $\nabla_{\kappa}$ and $(D l)_{\kappa}$ are equivalent (see [12]). For strongly inaccessible $\kappa$, the principle $(D l)_{\kappa}$ holds. In Corollary 2.13 we show that under suitable large cardinal hypotheses there is a forcing such that in the extension there is a regular non-strong limit cardinal $\kappa$ with $(D l)_{\kappa}$ and not $\nabla_{\kappa}$.

The use of the diamond $\nabla_{\kappa}$ or of the principle $(D l)_{\kappa}$ poses for many $\kappa$ no additional requirement. In Shelah [27] it is shown: If $\kappa$ is a successor and $\kappa^{<\kappa}=$ $\kappa>\aleph_{2}$ then $\diamond_{\kappa}$. On $(D l)_{\kappa}$, see Theorem 2.10.

It is not known whether $\kappa$-properness in the sense of Eisworth coincides with one of the various versions in the work of Rosłanowski and Shelah. The preservation theorems by Eisworth and by Rosłanowski and Shelah suggest that just replacing $\omega$ by $\kappa$ in the definition of "properness" does in general not suffice to preserve $\kappa^{+}$. The preservation of $\kappa^{+}$in iterations is usually guaranteed only under additional requirements on $\kappa$ and on the iterands.

There is an instructive example of a $(<\kappa)$-closed forcing that is not proper: We write $S_{\kappa}^{\lambda}=\{\alpha<\lambda: \operatorname{cf}(\alpha)=\kappa\}$. In the case of $2^{\mu}=\kappa$ for some $\mu<\kappa, 2^{\kappa}=\kappa^{+}$, a non-iterable $(<\kappa)$-closed forcing is the $\kappa^{++}$long iterated forcing $\mathbb{P}_{\text {unif }}$ adding a uniformisation to any colouring of a ladder system $\left\langle C_{\alpha}: \alpha \in \kappa^{+}, \operatorname{cf}(\alpha)=\kappa\right\rangle$, see [23, Appendix]. However, if $2^{\mu}=\kappa$, then no continuous ladder system on $S_{\kappa}^{\kappa^{+}}$has club uniformisation by [23, Theorem 3.6]. Thus $\left(\kappa^{+}\right)^{\mathbf{V}}$ is collapsed by $\mathbb{P}_{\text {unif }}$.

On the other hand Eisworth [6] shows for $\kappa=\aleph_{1}$ that for a stationary costationary $S \subseteq S_{\aleph_{1}}^{\aleph_{2}}$ the forcing adding a uniformisation to any colouring of a ladder system $\left\langle C_{\alpha}: \alpha \in S\right\rangle$ is consistent together with $\mathrm{CH}$.

For inaccessible cardinals $\kappa$, Friedman and Zdomskyy [7] and Friedman et al. [4, Section 5.2] define a version of club $\kappa$-Miller forcing. We present an equivalent version of the forcing and show that under $\kappa^{<\kappa}=\kappa>\omega$ a $\kappa$-version of Axiom A holds. For iteration with $\kappa$-support we use $(D l)_{\kappa}$.

\footnotetext{
${ }^{2}$ Heike Mildenberger thanks Stevo Todorcevic for pointing this out to her on the Logic Colloquium 2019 in Prague.
} 
Definition 2.1. Let $\kappa$ be a regular cardinal such that $\kappa^{<\kappa}=\kappa$. Conditions in the forcing order $\mathbb{Q}_{\kappa}^{\mathrm{a}}$ are trees $p \subseteq{ }^{\kappa>} \kappa$ with the following additional properties:

(1) (Club filter superperfectness) For any $s \in p$ there is an extension $t \unrhd s$ in $p$ such that

$$
\operatorname{osucc}_{p}(t):=\left\{\alpha \in \kappa: t^{\wedge}\langle\alpha\rangle \in p\right\} \text { is club in } \kappa .
$$

We require that each node has either only one direct successor or splits into a club.

We write for $t \in p$,

$$
\operatorname{succ}_{p}(t):=\left\{t^{\wedge}\langle\alpha\rangle \in \kappa: t^{\wedge}\langle\alpha\rangle \in p\right\}
$$

for the set of immediate $p$-successors of $t$.

(2) (Closure of splitting) For each increasing sequence of length $<\kappa$ of splitting nodes, the union of the nodes on the sequence is a splitting node of $p$ as well.

A condition $q$ is stronger than $p$ if $q \subseteq p$.

We remark that clauses (1) and (2) imply that any $p \in \mathbb{Q}_{\kappa}^{\mathrm{a}}$ is unbounded and has the following closure property:

(3) For every increasing sequence $\left\langle t_{i}: i<\lambda\right\rangle$ of length $\lambda<\kappa$ of nodes $t_{i} \in p \in$ $\mathbb{Q}_{\kappa}^{\mathrm{a}}$ we have that the limit of the sequence $\bigcup\left\{t_{i}: i<\lambda\right\}$ is also a node in $p$.

This clause is sometimes added to the definition, see e.g., [4, Definition 74], where the forcing is called $\mathbb{M I}_{\kappa}^{\text {Clubfilter }}$. Splitting into osucc-sets in the club filter is equivalent to splitting into club sets. The forcing $\mathbb{Q}_{\kappa}^{\mathrm{a}}$ is isomorphic to its version that works only with nodes that are strictly increasing elements of ${ }^{\kappa>} \kappa$. The forcing $\mathbb{Q}_{\kappa}^{\mathrm{a}}$ is isomorphic to a dense subset of $\mathbb{M I}_{\kappa}^{\text {Clubfilter }}$ and hence their regular open algebras coincide.

In [4, Proposition 77] it is shown that $\mathbb{Q}_{\kappa}^{\mathrm{a}}$ adds a $\kappa$-Cohen real, i.e., a generic for $\left(2^{<\kappa}, \unlhd\right)$. This is a sharp contrast to $\omega$-Miller forcing. The latter preserves $P$-points and hence does not add Cohen reals.

Definition 2.2 (See [10]). The $\kappa$-Sacks forcing $\mathbb{S}_{\kappa}$. Conditions in $\mathbb{S}_{\kappa}$ are perfect subtrees of $2^{<\kappa}$ such that each limit of a $(<\kappa)$-sequence of splitting nodes is a splitting node.

Interesting non-equivalent variants of $\kappa$-Sacks forcing were introduced by Landver in [14]. He replaces closure of splitting nodes by the requirement that the splitting nodes along each branch must be in a given normal filter over $\kappa$.

It is well-known (see e.g., $[7,10,17])$ that under $\kappa^{<\kappa}=\kappa$ the forcing order $\mathbb{Q}_{\kappa}^{a}$ fulfils a $\kappa$-version of Axiom A. We define $\leq_{\alpha}$ slightly differently from Friedman and Zdomskyy [7, Definition 2.2], so that the premise $\kappa^{<\kappa}=\kappa$ suffices for the fusion lemma for one iterand. However, in iterations the inaccessibility of $\kappa$ or the diamond is used in limit steps. See Discussion 2.7. We show that $(D l)_{\kappa}$ suffices.

Notation 2.3. We assume $\kappa^{<\kappa}=\kappa$. (This assumption is waived only in Section 4 and taken up again in Section 5.) We fix an enumeration $\left\langle\eta_{i}: i<\kappa\right\rangle$ of $\kappa^{<\kappa}$ such that $\eta_{i} \triangleleft \eta_{j}$ implies $i<j$.

DeFinition 2.4. For $\alpha<\kappa$ we let

$$
\operatorname{spl}_{\alpha}(p)=\{t \in \operatorname{spl}(p): \operatorname{otp}(\{s \subsetneq t: s \in \operatorname{spl}(p)\})=\alpha\}
$$


and

$$
\operatorname{cl}_{\alpha}(p):=\left\{s \in p:(\exists \gamma \leq \alpha)\left(\exists t \in \operatorname{spl}_{\gamma}(p)\right)(s \subseteq t) \wedge(\exists \beta \leq \alpha)\left(s=\eta_{\beta}\right)\right\} .
$$

We let $p \leq_{\alpha} q$ if $p \leq q$ and $\mathrm{cl}_{\alpha}(p)=\operatorname{cl}_{\alpha}(q)$.

Note $\left|\mathrm{cl}_{\alpha}(p)\right| \leq|\alpha|+\aleph_{0}<\kappa$.

LEMma 2.5. The forcing $\left(\mathbb{Q}_{\kappa}^{a}, \leq\right)$ together with the orders $\left(\leq_{\alpha}\right)_{\alpha<\kappa}$ fulfils the fusion lemma.

Proof. Let $\left\langle p_{\alpha}: \alpha<\kappa\right\rangle$ be a fusion sequence, i.e., $p_{\alpha} \leq_{\alpha} p_{\beta}$ for $\alpha<\beta$. Then $\emptyset \in \bigcap p_{\alpha}$. Now let $t \in \bigcap p_{\alpha}$. For $\alpha<\kappa$, let $\gamma_{\alpha}$ be maximal $\gamma$ such that $t \in \operatorname{cl}_{\gamma}\left(p_{\alpha}\right)$. By closure of splitting, the maximum exists. Since the $\left\langle p_{\alpha}: \alpha<\kappa\right\rangle$ are increasing in strength we have for $\alpha \leq \beta, \gamma_{\alpha} \geq \gamma_{\beta}$. Then let $\gamma$ be the minimum of the $\gamma_{\alpha}, \alpha<\kappa$. Let $\gamma<\alpha_{0}<\kappa$ be such that for any $\alpha \geq \alpha_{0}, t \notin \mathrm{cl}_{\gamma+1}\left(p_{\alpha}\right)$. Now we choose an increasing continuous sequence $\left\langle t_{\alpha}: \alpha_{0} \leq \alpha<\kappa\right\rangle$ such that $t_{\alpha}$ is a minimal node in $\operatorname{spl}_{\gamma+1}\left(p_{\alpha}\right)$ above $t_{\beta}, \alpha_{0} \leq \beta<\alpha$. There are club many $\alpha$ such that for any $t_{\beta}, \beta<\alpha$, there is some $i<\alpha$ such that $t_{\beta}=\eta_{i}$. We take the minimal limit element $\beta$ of such a club. Then by definition of $\leq_{\alpha}, t_{\beta}$ stays a $(\gamma+1)$-splitting node in all the $p_{\alpha}, \alpha<\kappa$. Its osucc-set in $\bigcap p_{\alpha}$ is a diagonal intersection of $\operatorname{osucc}_{p_{\alpha}}\left(t_{\beta}\right), \alpha<\kappa$.

It is clear that any $(<\kappa)$-limit of splitting nodes of $\bigcap p_{\alpha}$ is a splitting node in $\bigcap p_{\alpha}$.

Lemma 2.6. For every maximal antichain $A$ and every $\alpha$ and $p$ there is a $q \geq_{\alpha} p$ that is compatible with at most $\kappa$ members of the antichain.

Proof. For any $t \in \operatorname{spl}_{\alpha}(p)$ we find a club $C_{t} \subseteq \operatorname{osucc}_{p}(t)$ of $i$ such that for any $i \in C_{t}$ there is a condition $q_{t, i}$ with $p^{\left\langle t^{\wedge}\langle i\rangle\right\rangle} \leq q_{t, i}$. Since $A$ is a maximal antichain, there is some $a \in A$ such that there is some $r_{t, i} \geq q_{t, i}, a$. Now we let

$$
q:=\bigcup\left\{r_{t, i}: t \in \operatorname{spl}_{\alpha}(p), i \in C_{t}\right\} .
$$

The union $q$ is a condition and $q \geq_{\alpha} p$ and $q$ is compatible just with $\left|\operatorname{spl}_{\alpha}(p)\right| \cdot \kappa$ many elements of $A$.

In Section 4 we will see that the assumption $\kappa^{<\kappa}=\kappa$ is essential.

In summary $\left(\mathbb{Q}_{\kappa}^{\mathrm{a}}, \leq,\left(\leq_{\alpha}\right)_{\alpha<\kappa}\right)$ fulfils a $\kappa$-version of Axiom A and preserves $\kappa$.

If $2^{\kappa}=\kappa^{+}$then $\mathbb{Q}_{\kappa}^{\mathrm{a}}$ preserves cardinals strictly above $\kappa^{+}$.

DisCUSSION 2.7. As in the above, we restrict our attention to regular uncountable $\kappa$ with $\kappa^{<\kappa}=\kappa$. For $\kappa=\lambda^{+}>\aleph_{1}$ and $2^{\lambda}=\kappa$, by [27], $\diamond_{\kappa}$ holds. So only for $\kappa=\aleph_{1}$ and for regular non-strong limit cardinals $\kappa$, it is open whether $\kappa^{<\kappa}=\kappa$ is sufficient for preserving $\kappa^{+}$in iterations with support $\kappa$ of suitable $(<\kappa)$-closed tree forcing iterands like $\kappa$-Sacks forcing (see Definition 2.2) or club $\kappa$-Miller forcing (see Definition 2.1). For Sacks forcing, Kanamori asks this question in [10].

2.1. Fusion sequences for limits of cofinality $\kappa$. In this subsection we show that $\nabla_{\kappa}$ can be replaced by $(D l)_{\kappa}$ as a sufficient condition that the $\kappa$-support iteration of a tree forcing iterands with Axiom A preserves $\kappa^{+}$. This is a weak partial answer to Kanamori's question. First we show that $(D l)_{\kappa}$ can be strictly weaker than $\diamond_{\kappa}$. 
Definition 2.8 (See [21, 24, 25]).

(1) For a regular uncountable $\kappa$ and a stationary $S \subseteq \kappa$ we let $(D l)_{S}$ mean the following: There is a sequence $\bar{F}=\left\langle F_{\delta}: \delta \in S\right\rangle$ such that $F_{\delta} \subseteq{ }^{\delta} \delta$ is of cardinality $<\kappa$ and for every $f \in{ }^{\kappa} \kappa$ there are stationarily many $\delta \in S$ such that $f \uparrow \delta \in F_{\delta}$.

(2) We write $(D l)_{S}^{*}$ if in (1) we demand that the set $\left\{\delta \in S: f\left\lceil\delta \notin F_{\delta}\right\}\right.$ is not stationary.

FACT 2.9. We assume that $\kappa$ is regular and uncountable and $S \subseteq \kappa$ is stationary.

(1) If $\kappa$ is a successor cardinal then $(D l)_{S}$ is equivalent to $\diamond_{S}$ and $(D l)_{S}^{*}$ is equivalent to $\diamond_{S}^{*}$.

(2) If $\kappa$ is strongly inaccessible then $(\mathrm{Dl})_{S}^{*}$ holds.

(3) If $S_{1} \subseteq S_{2}$ are stationary in $\kappa$ then $(D l)_{S_{2}}$ implies $(D l)_{S_{1}}$ and the same for the diamond and both $*$-versions.

Proof. (1) Is proved by Kunen [12, Chapter III]. (2) We can let for $\delta \in S$, $F_{\delta}={ }^{\delta} \delta$, and we have that $\left\langle F_{\delta}: \delta \in S\right\rangle$ is a $(D l)_{S}^{*}$-sequence.

THEOREM 2.10. If $\mu$ is an uncountable strong limit cardinal and $\kappa^{<\kappa}=\kappa>\mu$ then for all but finitely many regular $\theta<\mu$ we have $(D l)_{\{\delta<\kappa: c f(\delta)=\theta\}}^{*}$.

Proof. See [25].

LEMMA 2.11.

(1) Assume that $\kappa$ is strongly inaccessible and $S \subseteq \kappa$ is stationary and $\mathbb{P}$ is a $\kappa$-c.c. forcing notion of cardinality $\kappa$. Then the following holds.

(a) In $\mathbf{V}^{\mathbb{P}},(D l)_{S}^{*}$ holds and hence $\kappa^{<\kappa}=\kappa$.

(b) If $\diamond_{S}$ holds in $\mathbf{V}$, then it holds also in $\mathbf{V}^{\mathbb{P}}$.

(2) If in addition $\mathbb{P}$ has the $\theta$-c.c. for some regular $\theta<\kappa$ then the following holds.

(a) In $\mathbf{V}^{\mathbb{P}}$ the cardinal $\kappa$ is weakly inaccessible.

(b) We assume yet in addition that $\theta \leq \mu<\kappa, \mu$ is regular, and

$$
\begin{gathered}
\mathbf{V} \models(\forall \alpha<\kappa)\left(c f(\alpha) \geq \mu \rightarrow|\alpha|^{<\theta}=|\alpha|\right) . \\
\text { Let } S=S_{\geq \mu}^{\kappa}:=\{\alpha<\kappa: c f(\alpha) \geq \mu\} . \text { If } \mathbf{V} \models \neg \nabla_{S}, \text { so } \mathbf{V}^{\mathbb{P}} \models \neg \nabla_{s} .
\end{gathered}
$$

Proof. (1)(a) Since $\mathbb{P}$ has the $\kappa$-cc., $S$ is also stationary in $\mathbf{V}^{\mathbb{P}}$. We choose an continuously increasing sequence $\left\langle\mathbb{P}_{\alpha}: \alpha<\kappa\right\rangle$ of subsets of $\mathbb{P}$ such that $\left|\mathbb{P}_{\alpha}\right|<\kappa$, $\mathbb{P}=\bigcup\left\{\mathbb{P}_{\alpha}: \alpha<\kappa\right\}$ and $\mathbb{P}_{\alpha} \leq_{\text {ic }} \mathbb{P}$, i.e., $\mathbb{P}_{\alpha}$ is an incompatibility preserving suborder of $\mathbb{P}$, incompatibility of two elements of $\mathbb{P}_{\alpha}$ within the order $\mathbb{P}_{\alpha}$ entails their incompatibilty in $\mathbb{P}$. Let $D_{\delta}$ be the set of canonical $\mathbb{P}_{\delta}$-names for functions in ${ }^{\delta} \delta$. Such a canonical name $\underset{\sim}{\tau}$ has, e.g., the form

$$
\underset{\sim}{\tau}=\left\{\left\langle p_{\gamma, \beta}^{\tau},\left\langle\gamma, f_{\sim}^{\tau}(\gamma, \beta)\right\rangle\right\rangle: \gamma<\delta, \beta<\beta_{\gamma}\right\},
$$

such that

(i) for $\gamma<\delta, \beta_{\gamma}<\kappa$.

(ii) for $\gamma<\delta, \beta<\beta_{\gamma}$, f $\tau(\gamma, \beta)<\delta$.

(iii) for $\gamma<\delta,\left\{p_{\gamma, \beta}^{\tau}: \beta<\beta_{\gamma}\right\}$ is a maximal antichain in $\mathbb{P}_{\delta}$. 
We let $P_{\delta}$ be a name for all evaluations of elements of $P_{\delta}$, so

$$
P_{\delta}=\left\{\left\langle 1_{\mathbb{P}}, \tau\right\rangle: \underset{\sim}{\tau} \text { is a canonical } \mathbb{P}_{\delta} \text {-name }\right\} .
$$

Since $\kappa$ is strongly inaccessibly and $\left(|\delta| \cdot\left|\mathbb{P}_{\delta}\right|\right)^{\left|\mathbb{P}_{\delta}\right|}<\kappa$, we have that for any $\delta<\kappa$, $\left|P_{\delta}\right|<\kappa$ and hence $\mathbb{P} \Vdash\left|P_{\delta}\right|<\kappa$.

Moreover, since $\mathbb{P}$ has the $\kappa$-c.c., every $\mathbb{P}$-name for an elements of ${ }^{\delta} \delta$ is a $\mathbb{P}_{\varepsilon}$-name for some $\varepsilon<\kappa$. We show that $\mathbb{P}$ forces that $\left\langle P_{\delta}: \delta \in S\right\rangle$ has the guessing property of a $(D l)_{S}^{*}$-sequence. Let $\underset{\sim}{ }$ be a canonical $\mathbb{P}$-name for a function from $\kappa$ to $\kappa$. We have to show that

$$
\mathbb{P} \Vdash\left\{\delta \in S: \underset{\sim}{\tau}\left\lceil\delta \in P_{\delta}\right\}\right. \text { is stationary. }
$$

We let

$$
C_{1}=\left\{\delta<\kappa:(\forall \alpha<\delta)\left\{p_{\alpha, \beta}^{\tau}: \beta<\beta_{\alpha}\right\} \subseteq \mathbb{P}_{\delta}\right\} .
$$

The set $C_{1}$ is club in $\kappa$. Let $\mathbb{P}$ force that $\underset{\tilde{V}}{C}$ be a $\mathbb{P}$-name for a club subset of $\kappa$. Since $\mathbb{P}$ has the $\kappa$-c.c., there is a club $C_{2} \in \tilde{\mathbf{V}}$, such that $\mathbb{P} \Vdash C_{2} \subseteq \underset{\sim}{C}$. Let $p \in \mathbb{P}$. Let $\delta \in S \cap C_{2} \cap C_{1}$. For $\gamma<\delta, \beta<\beta_{\gamma}, p_{\gamma, \beta}^{\tau} \Vdash \underset{\sim}{\tau}(\gamma)=f(\gamma, \beta)$. By the definition of $C_{1}$ and $P_{\delta}$, we have $\mathbb{P} \Vdash \tau \uparrow \delta \in P_{\delta}$. Hence (2.2) is proved.

(1)(b) We let $\mathbb{P}_{\alpha}, \alpha<\kappa$, be chosen as in the proof of (1)(a). Let $S \subseteq \kappa$ be stationary and let $\left\langle D_{\alpha}: \alpha \in S\right\rangle$ be a $\nabla_{S}$-sequence in $\mathbf{V}$. We assume that $\mathbb{P} \subseteq \kappa$. Then there is a club $C$ such that $\mathbb{P}_{\delta} \subseteq \delta$ for $\delta \in S \cap C$. Let $\varphi: \kappa \rightarrow \kappa \times(\kappa \times \kappa)$ be a bijection. By the $\kappa$-c.c, there is a club $C_{1} \subseteq C$ such that for $\delta \in C_{1}$,

$$
\begin{aligned}
& (\oplus)_{1} \varphi\lceil\delta \text { is onto } \delta \times(\delta \times \delta) \text { and } \\
& (\oplus)_{2}(\forall \beta<\delta)(\forall \gamma, \alpha<\kappa)(\langle\alpha,\langle\beta, \gamma\rangle\rangle \in \underset{\sim}{\tau} \rightarrow \alpha, \gamma<\delta) .
\end{aligned}
$$

Let for $\alpha \in S \cap C_{1}$,

$$
D_{\alpha}^{\prime}=\left\{\varphi(\beta): \beta \in D_{\alpha}\right\}
$$

We show that $\mathbb{P}$ forces that $\left\langle\left\langle 1_{\mathbb{P}},\left\langle\alpha, D_{\alpha}^{\prime}\right\rangle\right\rangle: \alpha \in S\right\rangle$ is a name for a diamond sequence on $S$ in $\mathbf{V}^{\mathbb{P}}$.

We let

$$
A=\left\{\varphi^{-1}(\langle\alpha,\langle\beta, \gamma\rangle\rangle):\langle\alpha,\langle\beta, \gamma\rangle\rangle \in \underset{\sim}{\tau}\right\} .
$$

Then there are stationarily many $\delta \in S \cap C_{1}$ such that $A \cap \delta=D_{\delta}$. By the choice of $C_{1}$ and of $D_{\delta}^{\prime}$, for any such $\delta$ we have

$$
\mathbb{P} \Vdash \underset{\sim}{\tau} \uparrow \delta=D_{\delta}^{\prime} .
$$

(2) (a) Any cardinal $\delta \in[\theta, \kappa]$ in $\mathbf{V}$ stays a cardinal in the extension. Since $\kappa$ is regular in the ground model, by the $\kappa$-c.c., it stays regular in the extension.

(2)(b) Now let $S$ be the special set as in the premise. A $\diamond_{S}^{-}$sequence is a $(D l)_{\kappa^{-}}$ sequence $\left\langle P_{\delta}: \delta<\kappa\right\rangle$ such that $\left|P_{\delta}\right| \leq \delta$. There is a function $f: \kappa \rightarrow \kappa$ such that for each $\delta<\kappa$, each $P_{\delta}$ has a $\mathbb{P}_{f(\delta)}$-name. By the $\theta$-c.c. and the assumption on cardinal expontiation, we have $(\forall \delta \in S)(f(\delta) \leq \delta)$. Let $\left\langle P_{\delta}: \delta \in S\right\rangle$ be a $\nabla_{S}^{-}$-sequence in $\mathbf{V}^{\mathbb{P}}$. Then $\mathbb{P} \Vdash\left|P_{\delta}\right| \leq|\delta|$. We let for $\delta \in S$,

$$
D_{\delta}=\left\{x \in{ }^{\delta} \delta \cap \mathbf{V}:\left(\exists p \in \mathbb{P}_{\delta}\right)\left(p \Vdash x \in P_{\delta}\right)\right\} .
$$


Since $\mathbb{P}$ has the $\theta$-c.c. and since $\mathbb{P} \Vdash\left|P_{\delta}\right| \leq \delta$, the set $D_{\delta}$ has size at most $|\delta|$. We show

$$
\left\langle D_{\delta}: \delta \in S\right\rangle
$$

is a $\diamond_{S}^{-}$-sequence in $\mathbf{V}$. Let $x \subseteq \kappa \in \mathbf{V}, C \subseteq \kappa$ be club in $\mathbf{V}$. By the $\kappa$-c.c., $C$ is also a club in $\mathbf{V}^{\mathbb{P}}$. Then for any $\delta<\kappa, x \cap \delta$ has a $\mathbb{P}_{\delta}$-name. By assumption, $\mathbb{P} \Vdash " ~ "\{\delta \in$ $C \cap S: x\left\lceil\delta \in P_{\delta}\right\} \neq \emptyset$." We pick $\delta \in C \cap S$ and $p \in \mathbb{P}$ such that $p \Vdash x \cap \delta \in P_{\delta}$. Since $\delta \in S$ we can choose $p \in \mathbb{P}_{\delta}$. Thus $x \cap \delta \in D_{\delta}$.

REMARK 2.12. Let $\kappa$ be strongly inaccessible. For any regular $\xi<\kappa$, the forcing adding $\kappa$ many $\xi$-Cohen reals, i.e.,

$$
\mathbb{P}=\left\{f:\left(\exists u \in[\kappa]^{<\xi}\right)(f: u \rightarrow\{0,1\})\right\},
$$

fulfils the premises of (1) and has the $\xi^{+}$-cc.

Finally we separate $\nabla_{S}$ from $(D l)_{S}$ for some stationary sets $S$.

COROLLARY 2.13. If there is a supercompact cardinal $\kappa$ and a measurable above $\kappa$, $\xi<\kappa$, then is consistent that $\kappa$ is strongly inaccessible and for any stationary $S \subseteq \kappa$, $(\mathrm{Dl})_{S}$ holds but $\diamond_{S}$ fails.

Under the same large cardinal assumptions there is a weakly inaccessible not strongly inaccessible cardinal $\kappa$ and there is are regular $\xi^{+}<\mu<\kappa$ such that for $S=\{\alpha<$ $\kappa: c f(\alpha) \geq \mu\}$ the principle $(D l)_{S}$ holds but the diamond $\nabla_{S}$ fails.

Proof. It suffices to force that $\kappa$ is strongly inaccessible and $\neg \nabla_{\kappa}$. Under the named large cardinal assumption there is such a forcing construction in unpublished work by Woodin. Golshani worked Woodin's construction out in [8]. Zeman [29] provides a lower bound on the consistency strength.

For the second part of the corollary, we consider Woodin's model as the ground model $\mathbf{V}$. The iterated modified Radin forcing that is based on a measure sequence forces (2.1).

We apply Lemma 2.11(1) and (2) to a forcing $\mathbb{P}$ as in Remark 2.12. Such a forcing destroys that $\kappa$ is a strong limit but preserves that $\kappa$ is weakly inaccessible. According to the second part of the lemma, $\nabla_{S}$ fails in $\mathbf{V}^{\mathbb{P}}$. Note that the notion of being a cardinal is not changed by $\mathbb{P}$ for cardinals of cofinality at least $\xi^{+}$.

This concludes our discussion of the principle $(D l)_{S}$.

Now we return to Miller and Sacks forcing.

DEFINITION 2.14.

(1) Let $\mathbb{P}_{\alpha}$ be the $\kappa$-support iteration of $\mathbb{Q}_{\kappa}^{\mathrm{a}}$. If $p, q \in \mathbb{P}_{\alpha}, \gamma<\kappa$, and $F \subseteq \operatorname{supp}(p)$ we write $q \geq_{F, \gamma} p$ if $\forall \beta \in F, q\left\lceil\beta \Vdash q(\beta) \leq_{\gamma} p(\beta)\right.$.

(2) We denote that support of a condition by $\operatorname{supp}(p)$.

Lemma 2.15 (Kanamori, proof of [10, Theorem 2.2]). Assume $(\mathrm{Dl})_{\kappa}$ and that $p \in \mathbb{P}_{\alpha}$ and $p \Vdash_{\mathbb{P}_{\alpha}} \tau \in$ On. Suppose also that $F \subseteq \operatorname{supp}(p)$ and $|F|<\kappa$, and that $\gamma<\kappa$. Then there is a $q \geq_{F, \gamma} p$ and an $x \subseteq$ On, $|x| \leq \kappa$, such that $q \Vdash \tau \in x$.

Proof. Sketch. We rework Kanamori [10, Theorem 2.2] and shows that it works with $(D l)_{\kappa}$ instead of $\nabla_{\kappa}$. The modification is: By $(<\kappa)$-closure, in the successor step, we can find a strengthening of all the $<\kappa$ many guesses offered by $(D l)_{\kappa}$ by strengthening successively, in $<\kappa$ substeps. 
As a routine conclusion of the technical lemma we get the following:

Theorem 2.16. Assume $\kappa^{<\kappa}=\kappa$ and $(D l)_{\kappa}$. Let $\delta$ be an ordinal and let $\mathbb{P}=$ $\left\langle\mathbb{P}_{\alpha}, \mathbb{Q}_{\beta}: \alpha \leq \delta, \beta<\delta\right\rangle$ be $a(\leq \kappa)$-support iteration of iterands $\mathbb{Q}_{\beta}=\mathbb{Q}_{\kappa}^{a}\left(\right.$ in $\left.\mathbf{V}^{\mathbb{P}} \beta\right)$. Then $\mathbb{P}_{\delta}$ has the following properties.

(1) $\mathbb{P}_{\delta}$ does not collapse $\kappa^{+}$.

(2) ( $\kappa$-properness) For any $N \prec H(\chi),|N|=\kappa$ with ${ }^{<\kappa} N \subseteq N$ and $\kappa, p, \mathbb{P}_{\delta} \in N$, and $p \in \mathbb{P}_{\delta}$ there is a stronger $\left(N, \mathbb{P}_{\delta}\right)$ generic condition $q$.

The same holds for any $\kappa$-support iteration of $\kappa$-Sacks forcing. We restate Kanamori's question:

Question 2.17. Can we replace $(D l)_{\kappa}$ by $\kappa^{<\kappa}=\kappa$ in Theorem 2.16?

§3. $\kappa^{++}$may be preserved or not. Here we are concerned with the preservation of $\kappa^{++}$. In this section we show that under the condition $2^{\kappa}=\kappa^{++}$the preservation of $\kappa^{++}$by $\mathbb{Q}_{\kappa}^{\mathrm{a}}$ is independent from ZFC.

Definition 3.1. Let $\mathbb{Q}$ be a forcing order and let $\lambda$ be a cardinal. $\operatorname{Ax}(\mathbb{Q},<\lambda)$ is the statement: For any set $\mathcal{D}$ of size $<\lambda$ of dense sets in $\mathbb{Q}$ there is a filter $G \subseteq \mathbb{Q}$ such that $(\forall D \in \mathcal{D})(G \cap D \neq \emptyset)$. Such a filter is called $\mathcal{D}$-generic.

Definition 3.2. An amoeba for $\mathbb{Q}_{\kappa}^{\mathrm{a}}$. See [13]. Let $\kappa>\aleph_{0}$ be an inaccessible cardinal. The amoeba for $\kappa$-Miller forcing, $\mathbb{Q}_{\kappa}^{\mathrm{a}, \text { amoeba }}$, is the following forcing order: Conditions are of the form $p=\left(t_{p}, T_{p}\right)$, where $T_{p} \in \mathbb{Q}_{\kappa}^{a}$ and $t_{p}$ is a subtree of $T_{p}$ that is $(<\kappa)$-closed in the following sense

(a) each increasing sequence of splitting nodes in $t_{p}$ of length $(<\kappa)$ is a splitting node of $t_{p}$ (hence $t_{p}$ has maximal nodes which are of successor length). We write

$$
\operatorname{term}\left(t_{p}\right)=\left\{s \in t_{p}:(\neg \exists t \triangleright s) t \in t_{p}\right\} .
$$

This set is called the set of terminal nodes of $t_{p}$. Since $t_{p}$ is $(<\kappa)$-closed, for each $s \in t_{p}$ there is a subset $F$ of $p^{\langle s\rangle}$ such that for each branch $b$ of $p^{\langle s\rangle}$ terminal $t \unrhd s, t \in b$.

(b) For each $t \in t_{p} \cap \operatorname{spl}(p) \backslash \operatorname{term}\left(t_{p}\right)$ we have osucc $t_{p}(t)$ is a closed initial subset of $\operatorname{osucc}_{T_{p}}(t)$.

The requirement (b) is used for $\bigcup\left\{t_{p}:\left(t_{p}, T_{p}\right) \in G\right\} \in \mathbb{Q}_{\kappa}^{\mathrm{a}}$. This is used in Theorem 3.4 (4)(b).

The forcing order is as follows: $\left(t_{p}, T_{p}\right) \leq\left(t_{q}, T_{q}\right)$ if $T_{q} \subseteq T_{p}, t_{q} \supseteq t_{p}$ and for each $\eta \in t_{p}, \operatorname{osucc}_{t}(\eta)$ is an initial segment of $\operatorname{osucc}_{t q}(\eta)$.

Results about $\mathbb{Q}_{\kappa}^{\text {a,amoeba }}$, for inaccessible $\kappa$, can be found in [13].

Definition 3.3. Now we define $\leq_{\alpha}$ for $\mathbb{Q}_{\kappa}^{\text {a,amoeba }}$ under the assumption $\kappa^{<\kappa}=\kappa$.

(1) Let $p=\left(t_{p}, T_{p}\right) \in \mathbb{Q}_{\kappa}^{\text {a,amoeba }}$.

We write

$$
\operatorname{term}\left(t_{p}\right)=\left\{s \in t_{p}:(\neg \exists t \triangleright s) t \in t_{p}\right\} .
$$

This set is called the set of terminal nodes of $t_{p}$. 
(2) Let $\alpha<\kappa$. We define

$$
\begin{aligned}
\operatorname{spl}_{\alpha}^{\text {amoeba }}(p)= & \left\{t \in \operatorname{spl}\left(T_{p}\right) \backslash t_{p}:\right. \\
& \left.\operatorname{otp}\left(\left\{s \in T_{p} \backslash t_{p}: s \subsetneq t \wedge s \in \operatorname{spl}\left(T_{p}\right)\right\}\right)=\alpha\right\} .
\end{aligned}
$$

(3) Let $\left\langle\eta_{\alpha}: \alpha<\kappa\right\rangle$ enumerate $\kappa^{<\kappa}=\kappa$ and let $\alpha<\kappa$.

$$
\begin{gathered}
\operatorname{cl}_{\alpha}^{\text {amoeba }}(p):=\left\{s \in T_{p}:(\exists \gamma \leq \alpha)\left(\exists t \in \operatorname{spl}_{\gamma}^{\text {amoeba }}(p)\right)(s \subseteq t)\right. \\
\left.\wedge(\exists \beta<\alpha)\left(s=\eta_{\beta}\right)\right\} .
\end{gathered}
$$

(4) For $\alpha<\kappa$ we let $p=\left(t_{p}, T_{p}\right) \leq_{\alpha} q=\left(t_{q}, T_{q}\right)$ if $p \leq q, t_{p}=t_{q}$ and $\mathrm{cl}_{\alpha}^{\mathrm{amoeba}}(p)=\mathrm{cl}_{\alpha}^{\mathrm{amoeba}}(q)$.

Now we state the positive part of the consistency result.

THeORem 3.4. Suppose that $\kappa>\omega, \kappa^{<\kappa}=\kappa$.

(1) $\operatorname{Ax}\left(\mathbb{Q}_{\kappa}^{a},<\kappa^{++}\right)$and $2^{\kappa}=\kappa^{++}$is consistent relative to ZFC.

(2) $\operatorname{Ax}\left(\mathbb{Q}_{\kappa}^{a},<\kappa^{++}\right)$implies that forcing with $\mathbb{Q}_{\kappa}^{a}$ does not collapse $\kappa^{++}$.

Now let $\kappa$ be inaccessible.

(3) $\operatorname{Ax}\left(\mathbb{Q}_{\kappa}^{\text {a,amoeba }},<\kappa^{++}\right)$and $2^{\kappa}=\kappa^{++}$is consistent relative to ZFC.

(4) $\operatorname{Ax}\left(\mathbb{Q}_{\kappa}^{a, \text { amoeba }},<\kappa^{++}\right)$implies
(a) forcing with $\mathbb{Q}_{\kappa}^{\text {a,amoeba }}$ does not collapse $\kappa^{++}$and
(b) forcing with $\mathbb{Q}_{\kappa}^{a}$ does not collapse $\kappa^{++}$.

Proof. (1) and (3). Over a ground model with $\kappa^{<\kappa}=\kappa, 2^{\kappa}=\kappa^{+}$and $\nabla_{\kappa}$, one performs the usual iteration with $(\leq \kappa)$-support as introduced in [10]. Any name for $\kappa^{+}$-many dense subsets of $\mathbb{Q}_{\kappa}^{\mathrm{a}}$ has an equivalent name that is hereditarily of size $<\kappa^{++}$, and hence there is a book-keeping device.

(2) Suppose that $\operatorname{Ax}\left(\mathbb{Q}_{\kappa}^{\mathrm{a}},<\kappa^{++}\right)$holds in $V$. Any $\mathbb{Q}_{\kappa}^{\mathrm{a}}$-name for a function $f: \mu \rightarrow$ $\kappa^{++}$for some $\mu<\kappa^{++}$is given by a name that consists of at most $\kappa^{+}$dense sets $\mathcal{D}$. $\operatorname{By} \operatorname{Ax}\left(\mathbb{Q}_{\kappa}^{\mathrm{a}},<\kappa^{++}\right)$there is a $\mathcal{D}$-generic filter in $V$. Thus $f$ cannot be onto.

(4) (a) is like (2).

(4)(b) Now for $\mathbb{Q}_{\kappa}^{\mathrm{a}}$. If $D \subseteq \mathbb{Q}_{\kappa}^{\mathrm{a}}$ is dense in $\mathbb{Q}_{\kappa}^{\mathrm{a}}$, then

$$
\operatorname{am}(D)=\left\{\left(t_{p}, T_{p}\right):\left(\forall s \in \operatorname{term}\left(t_{p}\right)\right)\left(T_{p}^{\langle s\rangle} \in D\right)\right\}
$$

is dense in $\mathbb{Q}_{\kappa}^{\text {a,amoeba }}$. Any $\mathbb{Q}_{\kappa}^{\text {a }}$-name $f$ for a function $f: \mu \rightarrow \kappa^{++}$for some $\mu<\kappa^{++}$ is given by a name that consists of at most $\kappa^{+}$dense sets $D_{\alpha}=\{p:(\exists \gamma) p \Vdash f(\check{\alpha})=$ $\check{\gamma}\}, \alpha<\mu$, put them into $\mathcal{D}$. $\operatorname{By} \operatorname{Ax}\left(\mathbb{Q}_{\kappa}^{\text {a,amoeba }},<\kappa^{++}\right)$there is a $\{\operatorname{am}(D): \tilde{D} \in \mathcal{D}\}$ generic filter $G$ in $V$. Now $T_{G}=\bigcup\left\{t_{p}: p \in G\right\}$ is a $\mathbb{Q}_{\kappa}^{\mathrm{a}}$-tree. We take any branch $b$ of $T_{G}$. Then

$$
\left\{T_{p}^{\langle s\rangle}: p=\left(t_{p}, T_{p}\right) \in G, s \in \operatorname{term}\left(t_{p}\right) \cap b\right\}
$$

is a $\mathcal{D}$-generic filter. Thus $f$ is determined in the ground model and hence cannot be a name for a function from $\mu$ onto $\kappa^{++}$.

Forcing with $\mathbb{Q}_{\kappa}^{\mathrm{a}}$ may collapse $2^{\kappa}$ to $\mathfrak{b}_{\kappa}$. Now we come to a complementary scenario: $\mathbb{Q}_{\kappa}^{\mathrm{a}}$ may collapse $2^{\kappa}$ to $\mathfrak{b}_{\kappa}$. Petr Simon showed that Sacks forcing collapses the continuum to the bounding number [28]. Here we prove, under additional hypotheses, a similar result for the higher case. Our result pertains to $\mathbb{Q}_{\kappa}^{\mathrm{a}}$. 
Definition 3.5. We recall the definition of three cardinal invariants at $\kappa$.

(1) The ideal of bounded subsets of $\kappa$ is $J_{\kappa}^{\mathrm{bd}}=[\kappa]^{<\kappa}$. The order of eventual domination is: $f \leq_{J_{\kappa}^{\text {bd }}} g$ if $\{\alpha: f(\alpha) \not \leq g(\alpha)\} \in J_{\kappa}^{\text {bd }}$. The bounding number at $\kappa$ is the following cardinal

$$
\mathfrak{b}_{\kappa}=\min \left\{|F|: F \subseteq \kappa^{\kappa} \wedge\left(\forall g \in \kappa^{\kappa}\right)(\exists f \in F)\left(f \mathbb{Z}_{J_{\kappa}^{\text {bd }}} g\right)\right\} .
$$

(2) The order of club-domination is defined on functions from $\kappa$ to $\kappa$ as follows: $f \leq_{\text {club }} g$ if $\{\alpha \in \kappa: f(\alpha) \leq g(\alpha)\}$ contains a club subset. The bounding number modulo a club at $\kappa$ is the following cardinal

$$
\mathfrak{b}_{\text {club }, \kappa}=\min \left\{|F|: F \subseteq \kappa^{\kappa} \wedge\left(\forall g \in \kappa^{\kappa}\right)(\exists f \in F)\left(f \mathbb{L}_{\text {club }} g\right)\right\} .
$$

By [5, Theorem 6], $\mathfrak{b}_{\text {club }, \kappa}=\mathfrak{b}_{\kappa}$ for any regular uncountable $\kappa$.

For any condition $p$ in a tree forcing, in particular for $\mathbb{Q}_{\kappa}^{a}$, there is a function mapping each node to the shortest splitting node above it. We work with this function for thinning out trees.

DEFINITION 3.6.

(1) We say $p \in \mathbb{Q}_{\kappa}^{\mathrm{a}}$ is nice if the following holds:

(a) For every $p \in \mathbb{Q}_{\kappa}^{\text {a }}$ for every $\eta \in p, \eta$ is a strictly increasing function.

(b) If $\eta \in \operatorname{spl}(p)$ then $\lg (\eta)$ is a limit ordinal.

(2) We fix an enumeration $\left\langle\eta_{\alpha}: \alpha<\kappa\right\rangle$ of ${ }^{\kappa>} \kappa$. Let $p \in \mathbb{Q}_{\kappa}^{\mathrm{a}}$. We define

$$
h_{p}:{ }^{\kappa>} \kappa \rightarrow{ }^{\kappa>} \kappa
$$

as follows: For $\eta=\eta_{\alpha} \in p \backslash \operatorname{spl}(p)$ we let $h_{p}(\eta)$ be the shortest $v$ such that $(\eta \hat{v} v \in \operatorname{spl}(p)$ and $\lg (\eta \hat{v} v) \geq \alpha)$.

For $\eta \notin p$ and for $\eta \in \operatorname{spl}(p)$, we let $h_{p}(\eta)=\emptyset$.

(3) Let $A \subseteq{ }^{\kappa>} \kappa . h:{ }^{\kappa>} \kappa \rightarrow{ }^{\kappa>} \kappa$ is called increasing on $A$ if for every $\eta \in A$ there is $(\varrho, \sigma) \in h(\eta)$ such that

$$
(\forall \alpha \in \operatorname{dom}(\eta))(\eta(\alpha)<\sigma) .
$$

The set of nice conditions in dense in the isomorphic copy of $\mathbb{Q}_{\kappa}^{\mathrm{a}}$ whose conditions contain only strictly increasing nodes. From now on we assume that any $p \in \mathbb{Q}_{\kappa}^{\mathrm{a}}$ contains only strictly increasing nodes.

Remark 3.7. If $p \in \mathbb{Q}_{\kappa}^{\mathrm{a}}$ is nice and $A=\left\{\eta^{\wedge}\langle\alpha\rangle: \eta \in \operatorname{spl}(p), \eta^{\wedge}\langle\alpha\rangle \in p\right\}$, then the function $h_{p}$ from Definition 3.6(2) is increasing on $A$.

We work with particular evasion numbers, defined as follows.

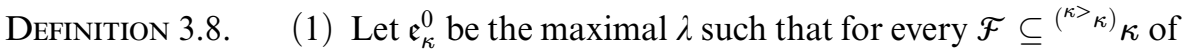
size $<\lambda$ and every everywhere strictly increasing $h:{ }^{\kappa>} \kappa \rightarrow{ }^{\kappa>} \kappa$ there is some $g:{ }^{\kappa>} \kappa \rightarrow \kappa$ with the following property:

For every $f \in \mathcal{F}$ and $\eta \in{ }^{\kappa>} \kappa$ there is a stationary set $S=S_{h, f, \eta}$ such that for any $\delta \in S$

$$
g\left(\eta^{\wedge}\langle\delta\rangle^{\wedge} h\left(\eta^{\wedge}\langle\delta\rangle\right)\right) \neq f\left(\eta^{\wedge}\langle\delta\rangle^{\wedge} h\left(\eta^{\wedge}\langle\delta\rangle\right)\right)
$$


(2) Actually we can work with a somewhat more specific variant of $\mathfrak{e}_{\kappa}^{0}$ : Let $\mathfrak{e}_{\kappa}^{1}$ be the maximal $\lambda$ such that for every nice $p \in \mathbb{Q}_{\kappa}^{\mathrm{a}}$ and $h=h_{p}$ and for every $\left.\mathcal{F} \subseteq{ }^{(\kappa>} \kappa\right) \kappa$ of size $<\lambda$ there is some $g:{ }^{\kappa>} \kappa \rightarrow \kappa$ with the following property:

For every $f \in \mathcal{F}$ and $\eta \in \operatorname{spl}(p)$ there is a stationary set $S=S_{h_{p}, f, \eta}$ such that for any $\delta \in S$

$$
\eta^{\wedge}\langle\delta\rangle \in p \wedge g\left(\eta^{\wedge}\langle\delta\rangle^{\wedge} h_{p}\left(\eta^{\wedge}\langle\delta\rangle\right)\right) \neq f\left(\eta^{\wedge}\langle\delta\rangle^{\wedge} h_{p}\left(\eta^{\wedge}\langle\delta\rangle\right)\right) .
$$

Lemma 3.9. Suppose that $\kappa=\kappa^{<\kappa}>\aleph_{0}$ and that $\mathbb{P}$ is the forcing adding $\lambda=\lambda^{\kappa}$ many Cohen reals with support $<\kappa$. Then in the extension we have

$$
\mathfrak{b}_{\kappa}=\mathfrak{b}_{\text {club }, \kappa}=\kappa^{+} \wedge \mathfrak{e}_{\kappa}^{0}=\mathfrak{e}_{\kappa}^{1}=\lambda .
$$

Proof. It is easy to see that the first $\kappa^{+}$Cohen reals form an unbounded family. Now we work in the forcing extension. Let $\mathbb{P}=\mathbb{P}_{\lambda}=\left\langle\mathbb{P}_{\alpha}, \mathbb{Q}_{\beta}: \beta<\lambda, \alpha \leq \lambda\right\rangle$ be the iteration, $\mathbb{Q}_{\alpha}=\left({ }^{\kappa>} \kappa, \triangleleft\right)$. Suppose $\mathcal{F}$ and $h$ are given as in the definition of $\mathfrak{e}_{\kappa}^{0}$. Since $\mathcal{F}$ is of size $<\lambda$, there is an initial segment $\mathbb{P}_{\alpha}$ of $\mathbb{P}_{\lambda}, \alpha<\lambda$ and a $p \in \mathbb{P}_{\alpha}$, such that $p$ forces that $\mathcal{F}$ and $h$ are $\mathbb{P}_{\alpha}$-names. We conceive Cohen reals as functions from ${ }^{\kappa>} \kappa \rightarrow \kappa$. Since $\kappa^{<\kappa}=\kappa$, this is possible. Now let $g=c_{\alpha}$ be the $\alpha$-th Cohen real.

Suppose for a contradiction that there is some $q \in \mathbb{P}_{\lambda}, q \geq p, \eta \in{ }^{\kappa>} \kappa, f \in \mathcal{F}$ and a name $\underset{\sim}{C}$ such that

$$
\begin{aligned}
& q \Vdash \underset{\sim}{C} \text { is club in } \kappa \wedge \\
& \quad(\forall \delta)\left(\delta \in \underset{\sim}{C} \rightarrow c_{\alpha}\left(\eta^{\wedge}\langle\delta\rangle^{\wedge} h\left(\eta^{\wedge}\langle\delta\rangle\right)\right)=f\left(\eta^{\wedge}\langle\delta\rangle^{\wedge} h\left(\eta^{\wedge}\langle\delta\rangle\right)\right)\right) .
\end{aligned}
$$

Now by induction on $n<\omega$ we choose $\delta_{-1}=0$ and increasing $q_{n} \geq q, q_{n} \in \mathbb{P}_{\lambda}$, and $\delta_{n}$ such that

$$
\begin{aligned}
& q_{n} \Vdash \delta_{n} \in \underset{\sim}{C} \wedge \delta_{n}>\delta_{n-1} \wedge \\
& c_{\alpha}\left(\eta^{\wedge}\left\langle\delta_{n}\right\rangle^{\wedge} h\left(\eta^{\wedge}\left\langle\delta_{n}\right\rangle\right)\right)=f\left(\eta^{\wedge}\left\langle\delta_{n}\right\rangle^{\wedge} h\left(\eta^{\wedge}\left\langle\delta_{n}\right\rangle\right)\right)
\end{aligned}
$$

and

$$
\left(\forall v \in \operatorname{dom}\left(q_{n}(\alpha)\right)\right)(\forall \sigma \in \operatorname{range}(v))\left(\sigma<\delta_{n+1}\right) .
$$

Then we let $r=\bigcup q_{n}$ and we let $\delta=\sup \delta_{n}$. Then

$$
r \Vdash \delta \in \underset{\sim}{C} .
$$

Since $h$ is an increasing function, we have

$$
\left.\eta^{\wedge}\langle\delta\rangle^{\wedge} h\left(\eta^{\wedge}\langle\delta\rangle\right)\right) \notin \operatorname{dom}(r(\alpha)) .
$$

Hence there is some $x \in \kappa$ such that

$$
\left.s=r \cup\left\{\left\langle\alpha,\left\langle\eta^{\wedge}\langle\delta\rangle^{\wedge} h\left(\eta^{\wedge}\langle\delta\rangle\right)\right), x\right\rangle\right\rangle\right\} \in \mathbb{P}_{\lambda}
$$

and

$$
x \neq f\left(\eta^{\wedge}\langle\delta\rangle^{\wedge} h\left(\eta^{\wedge}\langle\delta\rangle\right)\right) .
$$

So we reached a contradiction to the Assumption (3.1). For $\mathfrak{e}_{\kappa}^{1}$ we work with a name for a subset of $\operatorname{osucc}_{p}(\eta)$ in Equation (3.1). 
In preparation for the next theorem we recall nowhere distributivity and some notions for complete boolean algebras. Two forcings $\mathbb{P}_{1}, \mathbb{P}_{2}$ are said to be equivalent if their regular open algebras $\mathrm{RO}\left(\mathbb{P}_{i}\right)$ are isomorphic (for a definition of the regular open algebra of a poset, see, e.g., [9, Corollary 14.12]). Some forcings are characterised up to equivalence just by their density, their closure, and their collapsing behaviour. For a Boolean algebra $B$, we denote by $B^{+}=B \backslash\left\{0_{B}\right\}$.

Definition 3.10 ([1, Definition17]). Let $B$ be a Boolean algebra $\theta$, $\lambda$ cardinals. A collection $\mathcal{P} \subseteq \mathcal{P}\left(B^{+}\right)$is called a matrix if each member of $\mathcal{P}$ is a maximal disjoint subset of $B^{+}$.

A Boolean algebra $B$ is $(\theta, \lambda)$-distributive if for every matrix $\mathcal{P}=\left\{P_{\alpha}: \alpha \in \theta\right\}$ there is some maximal disjoint set $Q \subseteq B^{+}$such that for each $q \in Q$ and $\alpha \in \theta$, $\left|\left\{p \in P_{\alpha}: p \cap q \neq 0\right\}\right|<\lambda$.

A Boolean algebra is called $(\theta, \lambda)$-nowhere distributive if for every $x \in B^{+}$the algebra $B \nmid x=\{y \in B: y \leq x\}$ is not $(\theta, \lambda)$-distributive.

Lemma 3.11 ([1, Lemma 1.11]). If a Boolean algebra is $(\theta, \lambda)$-nowhere distributive then there are maximal antichains $\bar{p}^{\varepsilon}=\left\langle p_{\alpha}^{\varepsilon}: \alpha<\alpha_{\varepsilon}\right\rangle$ of $\mathbb{P}$ for $\varepsilon<\theta$ such that for every $p \in \mathbb{P}$ for some $\varepsilon<\theta$

$$
\left|\left\{\alpha<\alpha_{\varepsilon}: p \not \supset p_{\alpha}^{\varepsilon}\right\}\right| \geq \lambda .
$$

Definition 3.12. Let $B$ be a Boolean algebra. A subset $D \subseteq B^{+}$is called dense if $\left(\forall b \in B^{+}\right)(\exists d \in D)(d \leq b)$. The density of a Boolean algebra $B$ is the least size of a dense subset of $B$. A Boolean algebra $B$ has uniform density if for every $a \in B^{+}$, $B\lceil a$ has the same density. The density of a forcing order $(\mathbb{P},<)$ is the density of the regular open algebra $\mathrm{RO}(\mathbb{P})$.

From the following Lemma, only part (3) will be used later. Part (3) together with Lemma 3.11 gives an alternative proof of part (2).

Lemma 3.13. Let $\theta<\lambda$ be regular cardinals.

(1) [1, Theorem 1.15] Suppose that $\mathbb{P}$ has the following properties (a) to (c).

(a) $\mathbb{P}$ is a $(\theta, \lambda)$-nowhere distributive forcing notion,

(b) $\mathbb{P}$ has density $\lambda$,

(c) in case $\theta>\aleph_{0}, \mathbb{P}$ has $a(<\theta)$-complete dense subset $S$. The latter means: For

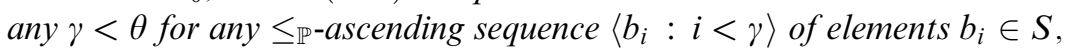
there is an upper bound $s \in S$, i.e., for any $i,\left(b_{i} \leq_{\mathbb{P}} s\right)$.

Then $\mathbb{P}$ is equivalent to Levy $(\theta, \lambda)$.

(2) [26] Under (a) and (b), $\mathbb{P}$ collapses $\lambda$ to $\theta$ (and may or may not collapse $\aleph_{0}$ ).

(3) Suppose that $\mathbb{P}$ has density $\lambda$ and that there are maximal antichains $P_{\varepsilon}=\left\langle p_{\varepsilon, \alpha}\right.$ : $\left.\alpha<\alpha_{\varepsilon}\right\rangle$ for $\varepsilon<\mathfrak{b}_{\text {club, },}$ as in Lemma 3.11. Then $\mathbb{P}$ collapses $\lambda$ to $\theta$.

Proof. (3) For completeness, we show such a name. We let for $\varepsilon<\mathfrak{b}_{\text {club, } \kappa}, P_{\varepsilon}$ be a maximal antichain such that for any $p \in \mathbb{Q}_{\kappa}^{\mathrm{a}}$, there is some $\varepsilon<\mathfrak{b}_{\mathrm{club}, \kappa}$ such that $\left|\left\{q \in P_{\varepsilon}: q \not \perp p\right\}\right| \geq \lambda$. We enumerate $\mathbb{Q}_{\kappa}^{\mathrm{a}}$ as $\left\langle p_{\alpha}: \alpha<\lambda\right\rangle$ such that each condition appears $\lambda$ times. For each $\varepsilon<\mathfrak{b}_{\text {club }, \kappa}$ we choose by induction on $\alpha<\lambda$ a pair $\left(q_{\varepsilon, \alpha}, r_{\varepsilon, \alpha}\right)$ such that

(a) $q_{\varepsilon, \alpha} \in\left\{s:\left(\exists r \in P_{\varepsilon}\right)(r \leq s)\right\}$ and for $\beta<\alpha, q_{\varepsilon, \alpha} \perp q_{\varepsilon, \beta}^{\prime}$. 
(b) If $\left|\left\{q \in P_{\varepsilon}: q \not \perp p_{\alpha}\right\}\right|=\lambda$ the we choose $r_{\varepsilon, \alpha} \geq p_{\alpha}, q_{\varepsilon, \alpha}$. Otherwise we let $r_{\varepsilon, \alpha}=q_{\varepsilon, \alpha}$.

This is easily done. Then we define a name $\underset{\sim}{\tau}$ of a function from $\mathfrak{b}_{\kappa}$ into $\lambda$ by letting for $\varepsilon \in \mathfrak{b}_{\kappa}$,

$$
\underset{\sim}{\tau}[G](\varepsilon)= \begin{cases}\operatorname{otp}\left\{\beta<\alpha: p_{\beta}=p_{\alpha}\right\}, & \text { if } r_{\varepsilon, \alpha} \in G(\text { this } \alpha \text { is unique }) ; \\ 0, & \text { else. }\end{cases}
$$

We show that $\underset{\sim}{\tau}$ is a name for a surjection from $\mathfrak{b}_{\kappa}$ to $\lambda$. Given a condition $p$ and $\alpha \in \lambda$, we first choose $\varepsilon<\mathfrak{b}_{\kappa}$ such that $\left|\left\{q \in \mathbb{P}_{\varepsilon}: q \not \perp p\right\}\right|=\lambda$ and enumerate $\left\{\beta<\lambda: p=p_{\beta}\right\}$ in increasing order via $\left\langle\beta_{\zeta}: \zeta<\lambda\right\rangle$.

Then we choose $\zeta=\alpha$ and have that

$$
r_{\varepsilon, \beta_{\alpha}} \Vdash \underset{\sim}{\tau}(\varepsilon)=\alpha .
$$

THEOREM 3.14. Suppose

(a) $\kappa=\kappa^{<\kappa}>\omega$ and

(b) $\mathfrak{e}_{\kappa}^{1}=\lambda=2^{\kappa}$.

Then $\mathbb{Q}_{\kappa}^{a}$ collapses $2^{\kappa}$ to $\mathfrak{b}_{\text {club, } \kappa}$.

Proof. It suffices to show that there are maximal antichains as in the premise of Lemma 3.11. Then Lemma 3.13(3) provides a name for a collapsing function.

$(\otimes)_{1}$ We fix an enumeration $\left\langle\eta_{i}: i<\kappa\right\rangle$ of $\kappa^{<\kappa}$ such that

(a) $\eta_{i} \triangleleft \eta_{j}$ implies $i<j$.

(b) For each $\alpha<\kappa, \lg \left(\eta_{\alpha}\right) \leq \alpha$.

(c) For each $\beta, \varepsilon<\kappa$ if $\eta^{\wedge}\langle\varepsilon\rangle=\eta_{\beta}$ then $\beta \geq \varepsilon$.

For $\eta \in{ }^{\kappa>} \kappa$, the index of $\eta$ is the minimal $\alpha$ such that $\eta=\eta_{\alpha}$.

$(\otimes)_{2}$ We fix a function $h_{*}: \kappa \rightarrow \kappa$ such that for any $\alpha<\kappa, h^{-1}[\{\alpha\}]$ is a stationary subset of $\kappa$.

$\left(\otimes_{3}\right)$ Now we define for nice conditions $p \in \mathbb{Q}_{\kappa}^{\mathrm{a}}$ three sets of nodes

$$
\Lambda_{p}^{1}=\{\eta \in \operatorname{spl}(p): \operatorname{otp}(\{\alpha<\lg (\eta): \eta\lceil\alpha \in \operatorname{spl}(p)\}) \text { is a limit ordinal }\} .
$$

If $\alpha<\lg (\eta)$ and $\eta\left\lceil\alpha \in \operatorname{spl}(p)\right.$ contains a limit order type, say $\alpha_{i}, i \in I$ converge to $\alpha_{\text {lim }}$, then it also contains $\eta \uparrow \alpha_{\text {lim }}$ since the latter must be a splitting point, so $\bigcup_{i \in I} \eta\left\lceil\alpha_{i}=\eta\right.$. So equivalently we can say that $\Lambda_{p}^{1}$ is the set of limits of splitting nodes.

We choose $\Lambda_{p}^{1}$ and not $\operatorname{spl}(p)$ for our thinning out procedures on splitting successors of limit splitting points that are called $\Lambda_{p}^{3}$. The point is that after the thinning out according to $(\otimes)_{4}$ we still have a condition, since we thin out at most the successor sets of splitting nodes at successor splitting levels.

$$
\begin{aligned}
& \Lambda_{p}^{2}=\left\{\eta^{\wedge}\langle\alpha\rangle: \eta \in \Lambda_{p}^{1}\right\}, \\
& \Lambda_{p}^{3}=\left\{\eta^{\wedge}\langle\alpha\rangle^{\wedge} h_{p}\left(\eta^{\wedge}\langle\alpha\rangle\right): \eta \in \Lambda_{p}^{1}, \eta^{\wedge}\langle\alpha\rangle \in \Lambda_{p}^{2}\right\} .
\end{aligned}
$$

For a nice $p$ and $\eta \in \Lambda^{1}(p)$ we have for any $\alpha$ such that $\eta^{\wedge}\langle\alpha\rangle \in p$, that $\eta^{\wedge}\langle\alpha\rangle \notin \operatorname{spl}(p)$ and $\eta^{\wedge}\langle\alpha\rangle h_{p}(\eta \hat{\wedge}\langle\alpha\rangle) \in \Lambda_{p}^{3}$. 
$(\otimes)_{4}$ For $p \in \mathbb{Q}_{\kappa}^{\mathrm{a}}, g:{ }^{\kappa>} \kappa \rightarrow \kappa$ we define $q=q(p, g)$ as the set of $\eta \in p$ such that

$$
\begin{aligned}
& (\forall \alpha<\lg (\eta))\left(\eta \left\lceil\alpha \in \Lambda_{p}^{3}\right.\right. \\
& \rightarrow \eta(\alpha)=\min \left\{\xi: \eta\left\lceil\alpha^{\wedge}\langle\xi\rangle \in p \wedge h_{*}(\xi)=g(\eta\lceil\alpha))\right\}\right) .
\end{aligned}
$$

Then $q(p, g)$ is a condition and $q(p, g) \geq p$.

If $p \in \mathbb{Q}_{\kappa}^{\mathrm{a}}, g$ is as above, and $q=q(p, g)$, then for any $r \geq q$, $\operatorname{spl}(r) \cap \Lambda_{p}^{3}=\emptyset$.

$(\otimes)_{5}$ Let $E$ be a club and $p \in \mathbb{Q}_{\kappa}^{\mathrm{a}}$. We say $p$ is suitable for $E$ if for any $\eta \in \operatorname{spl}(p)$ we have

$$
\left\{\delta<\kappa: \eta^{\wedge}\langle\delta\rangle \in p \wedge \lg \left(\eta^{\wedge}\langle\delta\rangle^{\wedge} h_{p}\left(\eta^{\wedge}\langle\delta\rangle\right)\right) \notin E\right\}
$$

is a stationary subset of $\kappa$.

$(\otimes)_{6}$ For $p, q \in \mathbb{Q}_{\kappa}^{\mathrm{a}}$ we define a function

$$
g_{p, q}:{ }^{\kappa>} \kappa \rightarrow \kappa
$$

as follows. If $v \in \Lambda_{p}^{3} \cap(q \backslash \operatorname{spl}(q))$, then we let

$$
g_{p, q}(v)=\min \left(h_{*}^{-1}[\{\xi\}]\right)
$$

for the unique $\xi<\kappa$ such that $v^{\wedge}\langle\xi\rangle \in q$. For all other $v$, we let $g_{p, q}(v)=0$.

$(\otimes)_{7}$ Let $E$ be club in $\kappa$ such that there is a $p$ suitable for $E$. We show: There is a maximal antichain $P_{E}=\left\langle q_{E, \alpha}: \alpha<2^{\kappa}\right\rangle$ with the following property: For every $p \in \mathbb{Q}_{\kappa}^{\mathrm{a}}$ that is suitable for $E$ there are $2^{\kappa}$ mutually incompatible stengthenings $q_{E, \alpha}>p, \alpha \in A$ for some $A \subseteq 2^{\kappa},|A|=2^{\kappa}$.

Proof: Let $\left\langle p_{E, \alpha}: \alpha<2^{\kappa}\right\rangle$ list the $p \in \mathbb{Q}_{\kappa}^{\mathrm{a}}$ that are suitable for $E$ each appearing $2^{\kappa}$ times. (In $(\otimes)_{9}$ we will see that there are such $p, E$.) We choose $q_{E, \alpha}$ be induction on $\alpha$ such that $p_{E, \alpha} \leq q_{E, \alpha}$ and $q_{E, \alpha} \in \mathbb{Q}_{\kappa}^{\text {a }}$ and $\beta<\alpha \rightarrow$ $q_{\beta} \perp q_{E, \alpha}$. In the end we take any maximal antichain containing $\left\{q_{E, \alpha}: \alpha<\right.$ $\left.2^{\kappa}\right\}$ as a subset. Since any $p$ that is suitable for $E$ appears at $2^{\kappa}$ many stages as $p_{E, \alpha}$, say at stages $\alpha \in A$, we have $2^{\kappa}$ many mutually incompatible $q_{E, \alpha}>$ $p=p_{E, \alpha}, \alpha \in A$.

Arriving at $\alpha$, for $\beta<\alpha$, the function

$$
f_{\beta}=g_{p_{E, \alpha}, q_{E, \beta}}:{ }^{\kappa>} \kappa \rightarrow \kappa
$$

is well defined. By our assumption on $\mathfrak{e}_{\kappa}^{1}=\lambda$, there is some $g$ for any $\eta \in$ $\operatorname{spl}\left(p_{\alpha}\right)$ such that $\operatorname{otp}\left(\left\{\alpha<\lg (\eta): \eta\left\lceil\alpha \in \operatorname{spl}\left(p_{\alpha}\right)\right\}\right)\right.$ is a limit ordinal +1 there are stationary many $\delta<\kappa$ such that $\eta^{\wedge}\langle\delta\rangle \in p_{\alpha}$ and

$$
f_{\beta}\left(\eta^{\wedge}\langle\delta\rangle^{\wedge} h_{p_{E, \alpha}}\left(\eta^{\wedge}\langle\alpha\rangle\right)\right) \neq g\left(\eta^{\wedge}\langle\delta\rangle h_{p_{E, \alpha}}\left(\eta^{\wedge}\langle\alpha\rangle\right)\right) .
$$

We let $q_{E, \alpha}=q\left(p_{E, \alpha}, g\right)$.

$(\otimes)_{8}$ We take a club-unbounded family $\left\langle f_{\varepsilon}^{*}: \varepsilon<\mathfrak{b}_{\kappa}\right\rangle$ such that the $f_{\varepsilon}^{*}$ are increasing functions.

For $\alpha<\mathfrak{b}_{\kappa}$, we let

$$
E_{\alpha}^{*}=\left\{\delta<\kappa: \delta \text { is a limit } \wedge(\forall \varepsilon<\delta)\left(f_{\alpha}^{*}(\varepsilon)<\delta\right)\right\} .
$$


$(\otimes)_{9}$ Claim: For every $p$ there is an $\varepsilon<\mathfrak{b}_{\kappa}$ such that $p$ is suitable of $E_{\varepsilon}^{*}$.

We let

$$
\begin{aligned}
E_{p}= & \{\delta<\kappa: \delta \text { is a limit ordinal and } \\
& \left.\left.(\forall \alpha, \beta<\delta)\left(\eta_{\alpha} \in p \rightarrow \eta_{\alpha} \hat{}\langle\beta\rangle, \eta_{\alpha}{ }^{\wedge} h_{p}\left(\eta_{\alpha}\right) \in\left\{\eta_{\beta}: \beta<\delta\right\}\right)\right)\right\} .
\end{aligned}
$$

Let $g_{p}(\varepsilon)$ be the $\varepsilon+7$-th member of $E_{p}$. Pick $\alpha_{p}=\alpha<\mathfrak{b}_{\text {club, } \kappa}$ such that

$$
\left\{\varepsilon<\kappa: g_{p}(\varepsilon)<f_{\alpha}^{*}(\varepsilon)\right\}
$$

is a stationary subset of $\kappa$.

Now we show that $p$ is suitable for $E_{\alpha}^{*}$. We have to show: For any $\eta \in \operatorname{spl}(p)$ we have

$$
\left\{\delta<\kappa: \eta^{\wedge}\langle\delta\rangle \in p \wedge \lg \left(\eta^{\wedge}\langle\delta\rangle^{\wedge} h_{p}\left(\eta^{\wedge}\langle\delta\rangle\right)\right) \notin E_{\alpha}^{*}\right\}
$$

is a stationary subset of $\kappa$.

Let $\eta=\eta_{\beta} \in \operatorname{spl}(p)$. By our choice of $\alpha$,

$$
A=\left\{\varepsilon>\beta: \varepsilon \in E_{p} \cap E_{\alpha}^{*} \wedge g_{p}(\varepsilon)<f_{\alpha}^{*}(\varepsilon)\right\}
$$

is a stationary subset of $\kappa$. If $\varepsilon \in A$, then $\zeta=\min \left\{\gamma \in E_{p}: \gamma>\varepsilon\right\} \in E_{p}$ and $\zeta<g_{p}(\varepsilon)$, since the latter is the $\varepsilon+7$-th member of $E_{p}$. By the defition of $E_{p}$, $\varepsilon<\zeta$ and $\zeta \in E_{p}$ implies that $\eta^{\hat{\langle}}\langle\varepsilon\rangle \subseteq\left\{\eta_{\gamma}: \gamma<\zeta\right\}$, and hence

$$
\eta^{\wedge}\langle\varepsilon\rangle^{\wedge} h\left(\eta^{\wedge}\langle\varepsilon\rangle\right) \in\left\{\eta_{\gamma}: \gamma<\zeta\right\} .
$$

If $\varepsilon \in A$ then

$$
\varepsilon<\zeta<g_{p}(\varepsilon)<f_{\alpha}^{*}(\varepsilon)<\operatorname{succ}_{E_{\alpha}^{*}}(\varepsilon) .
$$

Recall, we stipulated properties $(\mathrm{a})$ and $(\mathrm{b})$ of $(\otimes)_{1}$ on the enumeration. Hence $\lg \left(\eta_{\gamma}\right) \leq \gamma$ and the index of $\eta^{\wedge}\langle\varepsilon\rangle$ is larger than $\varepsilon$. By the definition of $h_{p}$ we get

$$
\operatorname{succ}_{E_{\alpha}^{*}}(\varepsilon)>\lg \left(\eta^{\wedge}\langle\varepsilon\rangle^{\wedge} h_{p}\left(\eta^{\wedge}\langle\varepsilon\rangle\right)\right) \geq \zeta .
$$

Hence $h_{p}\left(\eta^{\wedge}\langle\varepsilon\rangle\right)$ is longer than $\varepsilon$. So for any $\varepsilon \in A$ we have

$$
\lg \left(\eta^{\wedge}\langle\varepsilon\rangle^{\wedge} h_{*}\left(\eta^{\wedge}\langle\varepsilon\rangle\right)\right) \notin E_{\alpha}^{*},
$$

and the claim is proved.

Now we show that we now have established the premise of Lemma 3.11. We start with $\alpha_{0}<\kappa^{+}$such that there is at all a $p$ that is suitable for $E_{\alpha_{0}}^{*}$. Hence $p$ is suitable also for $E_{\alpha}^{*}, \alpha \geq \alpha_{0}$. We take for $\alpha \in\left[\alpha_{0}, \kappa^{+}\right)$an antichain $P_{E_{\alpha}^{*}}$ as in $(\otimes)_{7}$. For any $p \in \mathbb{Q}_{\kappa}^{\text {a }}$ there is a $\beta \geq \alpha_{0}$ such that $p$ is suitable for $E_{\beta}^{*}$. Hence by $(\otimes)_{7}$ the set $\left\{q \in P_{E_{\beta}^{*}}: q \not \perp p\right\}$ has size $\lambda$.

COROLlary 3.15. It is consistent relative to ZFC that $\kappa^{<\kappa}=\kappa>\omega, \mathfrak{b}_{\kappa}=\kappa^{+}$, $2^{\kappa}>\kappa^{+}$and $\mathbb{Q}_{\kappa}^{a}$ collapses $2^{\kappa}$ to $\mathfrak{b}_{\kappa}$.

We do not know whether our conditions on the evasion numbers are necessary.

QUESTION 3.16. Does $\mathbb{Q}_{\kappa}^{a}$ collapse $2^{\kappa}$ to $\mathfrak{b}_{\kappa}$ just under the condition $2^{\kappa}=\kappa^{++}>\mathfrak{b}_{\kappa}$ ? 
§4. Waiving $\kappa^{<\kappa}=\kappa$. In this section we collect mathematical reasons for the fact that in higher tree forcings the condition $\kappa^{<\kappa}=\kappa$ is very common. We show that under $\kappa^{<\kappa}>\kappa$, the forcing $\mathbb{Q}_{\kappa}^{\mathrm{a}}$ collapses $\kappa^{<\kappa}$ to $\kappa$. We focus on regular $\kappa$. This proof of the next theorem is quite complicated and was found before the more general Theorem 4.2. We let it stand because the name is different in particular in the choice of the lengths of trunks that are actually evaluated. If $\theta>\omega$ is regular, then then in the next theorem we give a much simpler name of a collapse of $\kappa^{\theta}$ to $\kappa$.

THEOREM 4.1. If c $f(\kappa)=\kappa=\lambda^{+}$and $\kappa \geq \theta^{++}$, and $\kappa^{\theta}>\kappa$, then $\mathbb{Q}_{\kappa}^{a}$ collapses $\kappa^{\theta}$.

Proof. By [22, Lemma 4.4.(3)] for $\lambda^{+}=\kappa$ there is a sequence $\bar{C}$ and there are $T, S_{i}, i<\lambda$, with the following properties:

(1) $T=\{\alpha \in \kappa: \operatorname{cf}(\alpha) \leq \theta\}$ and

(2) $T$ is the union of stationary sets $S_{i}, i<\lambda$, that have the following square property:

(i) There is $\bar{C}^{i}=\left\langle C_{\alpha}^{i}: \alpha \in S_{i}\right\rangle$,

(ii) $C_{\alpha}^{i}$ is a closed subset of $\alpha$, not necessarily cofinal in $\alpha$, however, if $\alpha$ is a limit ordinal, then $C_{\alpha}^{i}$ is cofinal in $\alpha, C_{\alpha}^{i} \subseteq T \cap \alpha$ and $\operatorname{otp}\left(C_{\alpha}^{i}\right) \leq \theta$, and

(iii) for $\alpha \in S_{i}$, for any $\beta \in C_{\alpha}^{i}$, then $\beta \in S_{i}$ and $C_{\beta}^{i}=C_{\alpha}^{i} \cap \beta$.

Again, we choose in the ground model a function $h_{*}: \kappa \rightarrow \kappa$ such that for each $\varepsilon \in \kappa$, there are stationarily many $\alpha$ with $h_{*}(\alpha)=\varepsilon$.

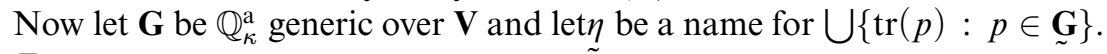

First step.

There is a pair $(i, E)$ such that

(a) $E$ is club in $\kappa$,

(b) $i<\kappa$, and

(c) for every club $C$ of $\kappa$ there are stationarily many $\alpha \in S_{i}$ such that $C \cap E \cap$ $C_{\alpha}^{i}=E \cap C_{\alpha}^{i}$ and $\operatorname{otp}\left(E \cap C_{\alpha}^{i}\right)=\theta$.

A proof is given, e.g., in [11, Fact 3.7].

Let $S=S_{i} \cap E$ and $C_{\alpha}=C_{\alpha}^{i} \cap E$ for $\alpha \in S_{i}$. Let

$$
S^{\prime}=\left\{\alpha \in S: \operatorname{otp}\left(C_{\alpha}\right)=\theta\right\} .
$$

For $\alpha \in S^{\prime}$ let $\langle\gamma(\alpha, j): j<\theta\rangle$ list $C_{\alpha}$ in increasing order.

Now for each $\alpha \in S^{\prime}$ we define a $\mathbb{P}$-name $\eta_{\alpha}$ of a function from $\theta$ into $\kappa$ by letting for $j \in \theta$ and $\alpha \in S^{\prime}$

$$
{\underset{\sim}{\eta}}_{\alpha}(j)=h_{*}(\underset{\sim}{\eta}(\gamma(\alpha, j))) \text {. }
$$

Second step.

We show: If $p \in \mathbb{P}$ and $v \in{ }^{\theta} \kappa$ then for some pair $(\alpha, q)$ we have

$(\alpha) \alpha \in S^{\prime}$,

( $\beta) q \geq p$, and

$(\gamma) q \Vdash_{\mathbb{P}}{\underset{\sim}{\alpha}}_{\alpha}=v$.

This is done as follows: For every $p$ and $v$ by induction on $\beta<\kappa$ we choose $\varrho_{\beta}$ such that

(a) $\varrho_{\beta} \in \operatorname{spl}(p)$,

(b) for $\beta<\gamma<\kappa, \varrho_{\beta} \unlhd \varrho_{\gamma}$, 
(c) at limits $\delta<\kappa, \varrho_{\delta}=\bigcup_{\beta<\delta} \varrho_{\beta}$, and

(d) if $\gamma=\beta+1$ and $\beta \in S$, and $\lg \left(\varrho_{\beta}\right)=\beta$, then $g\left(\varrho_{\gamma}(\beta)\right)=v\left(\operatorname{otp}\left(C_{\beta}\right)\right)$.

Note, for $\beta<\alpha, \alpha \in S_{i}, \gamma(\alpha, j)=\beta$ :

$$
\operatorname{otp}\left(C_{\beta}\right)=\operatorname{otp}\left(C_{\alpha} \cap \beta\right)=j .
$$

So $\left\langle\lg \left(\varrho_{\beta}\right): \beta<\kappa\right\rangle$ is increasing and continuous in $\kappa$. Hence $C^{\prime}=\left\{\lg \left(\varrho_{\beta}\right): \beta<\right.$ $\kappa\}$ is a club in $\kappa$ and there is $\alpha$ in $S^{\prime}$ such that $C_{\alpha} \subseteq C^{\prime}$ and $\operatorname{cf}(\alpha)=\theta$. By item (d) and Equation (4.2), we have for any $j<\theta, \gamma(\alpha, j) \in C_{\alpha}$, if $\gamma(\alpha, j)=\beta<\alpha$ then

$$
h_{*}(\underset{\sim}{\eta}(\gamma(\alpha, j)))=h_{*}\left(\rho_{\beta+1}(\beta)\right)=v(j) .
$$

Since $p^{[\varrho \alpha]}$ determines $\underset{\sim}{\eta}\lceil\alpha$, we have

$$
p^{\left[\varrho_{\alpha}\right]} \Vdash \underset{\sim}{\eta_{\alpha}}(j)=h_{*}\left(\varrho_{\alpha}(\gamma(\alpha, j))=v(j) .\right.
$$

As $\left|S^{\prime}\right|=\kappa$, we see that forcing with $\mathbb{P}$ collapses $\kappa^{\theta}$ to $\kappa$.

THEOREM 4.2.

(1) If $\kappa=c f(\kappa)>\theta=c f(\theta)>\omega$ and $\kappa^{\theta}>\kappa$, then forcing with $\mathbb{Q}_{\kappa}^{a}$ collapses $\kappa^{\theta}$ to $\kappa$.

(2) If $\kappa=\aleph_{1}$ and $2^{\omega}>\aleph_{1}$, then forcing with $\mathbb{Q}_{\kappa}^{a}$ collapses $2^{\omega}$ to $\omega_{1}$.

Proof. (1) On ${ }^{\theta} \kappa$ we define an equivalence relation $E$ as follows:

$$
\varrho E \sigma \text { if }\{\alpha<\theta: \varrho(\alpha)=\sigma(\alpha)\} \text { contains a club in } \theta .
$$

By Solovay's theorem, that there are $\theta$ mutually disjoint stationary subsets of $\theta$, the relation $E$ has $\kappa^{\theta}$ classes. Let $\left\langle\varrho_{\zeta}: \zeta<\kappa^{\theta}\right\rangle$ list a set of representatives without any repetitions.

Again we choose and fix a function $h_{*}$ from $\kappa$ to $\kappa$ such that for each $\alpha \in \kappa$, $h_{*}^{-1}[\{\alpha\}]$ is a stationary subset of $\kappa$. We let $\eta$ be a name for the $\mathbb{Q}_{\kappa}^{\mathrm{a}}$-generic $\kappa$-real. Now we define a name for a function

$$
\underset{\sim}{\tau}:\{\delta<\kappa: \operatorname{cf}(\delta)=\theta\} \rightarrow \kappa^{\theta},
$$

by letting $\underset{\sim}{\tau}(\delta)$ be the minimal $\zeta \in \kappa^{\theta}$ such that there is an increasing continuous sequence $\left\langle\alpha_{i}: i<\theta\right\rangle$ converging to $\delta$ such that

$$
\left\langle h_{*}\left(\underset{\sim}{\eta}\left(\alpha_{i}\right)\right): i<\theta\right\rangle E \varrho_{\zeta} .
$$

The particular choice of $\left\langle\alpha_{i}: i<\theta\right\rangle$ does not matter, since for any two such sequences that are club many $i<\theta$ on which they coincide. We prove that $\mathbb{Q}_{\kappa}^{\mathrm{a}}$ forces that $\tau$ is surjective. Given $\zeta \in \kappa^{\theta}$ we choose by induction on $i<\theta$, a node $\eta_{i} \in \operatorname{spl}(p)$ and ordinals $\alpha_{i} \in \kappa, \beta_{i} \in \kappa$, with the following properties:

(a) $\eta_{0}=\operatorname{tr}(p)$

(b) $\lg \left(\eta_{i}\right)=\alpha_{i}$,

(c) $\eta_{i+1} \triangleright \eta_{i} \hat{\langle}\left\langle\beta_{i}\right\rangle\left(\right.$ - so $\left.\eta_{i+1}\left(\alpha_{i}\right)=\beta_{i}-\right)$ and $\beta_{i} \in \operatorname{osucc}_{p}\left(\eta_{i}\right)$ is chosen such that $h_{*}\left(\beta_{i}\right)=\varrho\left(\alpha_{i}\right)$, and

(d) $\eta_{\delta}=\bigcup\left\{\eta_{i}: i<\delta\right\}$ for limit $\delta \leq \theta$.

In the end we let $q=p^{\left\langle\eta_{\theta}\right\rangle}$ and $\delta=\bigcup\left\{\alpha_{i}: i<\theta\right\}$ have

$$
q \Vdash\left\langle h_{*}\left(\underset{\sim}{\eta}\left(\beta_{i}\right)\right): i<\theta\right\rangle E \varrho_{\zeta} \wedge \lim _{i \rightarrow \theta}\left(\alpha_{i}\right)=\delta \wedge \underset{\sim}{\tau}(\delta)=\zeta .
$$


(2) Now for the case of $\kappa=\aleph_{1}$. For any limit ordinal $\delta \in \kappa$ we choose an increasing sequence $\overline{\alpha_{\delta}}=\left\langle\alpha_{\delta, n}: n<\omega\right\rangle$ that converges $\delta$. Again we let $\eta$ be a name for the generic branch. We let $S=\{\delta<\kappa: \delta$ limit ordinal $\}$. We let

$$
\begin{aligned}
&{\underset{\sim}{w}}_{\delta}=\{(n, i): n<\omega \wedge i \in S \wedge \\
& \underset{\sim}{\eta}(i) \in\left[\alpha_{\delta, n}, \alpha_{\delta, n+1}\right) \wedge(\forall j<i) \underset{\sim}{\left.\left(\underset{\sim}{\eta}(j)<\alpha_{\delta, n}\right)\right\} .}
\end{aligned}
$$

We define an equivalence relation $E$ on ${ }^{\omega} \omega$ :

$$
\varrho E \sigma \text { if }(\exists n \in \mathbb{Z})(\forall m)(\varrho(m)=\sigma(m+n)) .
$$

We let $\left\langle\varrho_{\zeta}: \zeta \in 2^{\omega}\right\rangle$ list a system of representatives without repetitions.

Recall: $\kappa=\omega_{1}$ and we let $h_{*}: \omega_{1} \rightarrow \omega_{1}$ be such that for any $\xi \in \omega_{1}, h_{*}^{-1}[\{\xi\}]$ is stationary in $\omega_{1}$.

Now we define a name ${\underset{\sim}{\delta}}_{\delta}$ as follows: $p \Vdash \underset{\sim}{v_{\delta}}(n)=\gamma$ if for some $m \in \omega$ and $i \in S$,

$$
\begin{aligned}
& p \Vdash\left((m, i) \in w_{\delta} \wedge\right. \\
& \quad n=\left|\left\{m^{\prime} \leq m:\left(\exists j<\omega_{1}\right)\left(m^{\prime}, j\right) \in w_{\delta}\right\}\right| \wedge \\
& \left.\quad h_{*}(i)=\gamma\right) .
\end{aligned}
$$

We show that

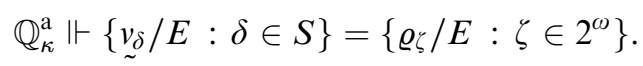

This will finish the proof, since then for any $\mathbb{Q}_{\kappa}^{\mathrm{a}}$-generic filter $G$ over $\mathbf{V}$, in $\mathbf{V}[G]$ the map on $S$ such that

$$
\delta \mapsto \zeta \text { for the } \zeta \text { with } \underset{\sim}{v_{\delta}}[G] E \varrho_{\zeta}
$$

is a collapsing function from $S$ onto $2^{\omega}$. Since $\kappa$ is uncountable, also in the extension $|S|=\omega_{1}$.

Heading for (4.5), given $p, \varrho$, we show that there are a stronger condition $q$ and some $\delta \in S$ such that $q \Vdash v_{\delta} E \varrho$.

We choose an increasing continuous sequence $\left\langle M_{\alpha}: \alpha<\omega_{1}\right\rangle$ of countable elementary submodels of $(H(\chi), \in,<)$ such that $\left\langle\overline{\alpha_{\delta}}: \delta \in S\right\rangle, \omega_{1}, \varrho, p \in M_{0}$.

We take $\delta \in S$ such that $M_{\delta} \cap \omega_{1}=\delta$. Since $\left\langle M_{\alpha}: \alpha<\omega_{1}\right\rangle$ is increasing and continuous, such a $\delta \in S$ exists.

The aim is to show that there is some node $\eta \in p$ such that

$$
p^{\langle\eta\rangle} \Vdash v_{\delta} E \varrho .
$$

We let:

$$
w^{(\omega)}=\left\{n<\omega:\left(\exists^{\geq \omega} \alpha<\omega_{1}\right)\left(M_{\alpha} \cap \omega_{1} \in\left[\alpha_{\delta, n}, \alpha_{\delta, n+1}\right)\right)\right\} .
$$

We prove that $w^{(\omega)}$ is infinite. Suppose that $n_{0}$ is given. Then there is some $\beta<\delta$ such that $M_{\beta} \cap \omega_{1} \geq \alpha_{\delta, n_{0}}$ and moreover $\beta+\omega<\delta$, since $M_{\delta} \prec(H(\chi), \in$, $<)$ and hence $M_{\delta}$ with $\omega, \beta \in M_{\delta}$ fulfils the replacement scheme for the function $\langle(n, \beta+n): n<\omega\rangle$. So $M_{\beta+\omega} \cap \omega_{1}<\alpha_{\delta, n_{1}}$ for some $n_{0}<n_{1}<\omega$. Hence all the $M_{\beta+n} \cap \omega_{1}, n \leq \omega$, are in the interval $\left[\alpha_{\delta, n_{0}}, \alpha_{\delta, n_{1}}\right)$. The latter interval is the union of $\left[\alpha_{\delta, n} \alpha_{\delta, n+1}\right), n_{0} \leq n<n_{1}$. Hence one of these contains $M_{\beta+m} \cap \omega_{1}$ for $m$ from an infinite set. 
Let $n_{0}<n_{1} \ldots$ list $w^{(\omega)}$ in increasing order.

By induction on $\ell<\omega$ we choose an increasing sequence of splitting nodes $\eta_{\ell} \in$ $\operatorname{spl}(p)$ and $\varepsilon_{\ell} \in \kappa$ such that such that

$(\alpha) \eta_{\ell} \triangleleft \eta_{\ell+1}$,

$(\beta)$ for $\ell \geq 0, i_{\ell}=\lg \left(\eta_{\ell}\right)=M_{\varepsilon_{\ell}} \cap \omega_{1} \in\left[\alpha_{\delta, n_{\ell}}, \alpha_{\delta, n_{\ell+1}}\right)$,

$(\gamma) \eta_{\ell}$ is a $\omega$-limit of splitting nodes and range $\left(\eta_{\ell}\right) \subseteq M_{\varepsilon_{\ell}} \cap \omega_{1}$, and

$(\delta) \eta_{\ell+1}\left(\lg \left(\eta_{\ell}\right) \in h_{*}^{-1}[\{\varrho(\ell)]\right.$.

To find $\eta_{0}$ and $\varepsilon_{0}$ we apply the second half of the successor step to $\operatorname{rt}(p)$ and $M_{0}$.

We carry out the successor step: Suppose $\eta_{\ell}$ and $\varepsilon_{\ell}$ are chosen. and $\operatorname{dom}\left(\eta_{\ell}\right)=$ $i_{\ell}=M_{\varepsilon_{\ell}} \cap \omega_{1}$ has range contained in $M_{\varepsilon_{\ell}} \cap \omega_{1}$. Hence

$$
\left(\forall j<i_{\ell}\right)\left(\eta_{\ell}(j)<\alpha_{\delta, n_{\ell}+1}\right) .
$$

We choose $\alpha$ such that $\eta_{\ell}\langle\alpha\rangle \in p$ and we choose a preliminary $\varepsilon_{\ell+1}^{\prime}$ with the following properties: We let $\varepsilon_{\ell+1}^{\prime} \geq \varepsilon_{\ell}+1$ such that $M_{\varepsilon_{\ell+1}^{\prime}} \cap \omega_{1} \in\left[\alpha_{n_{\ell+1}}, \alpha_{n_{\ell+1}+1}\right)$. Since $\eta_{\ell}$ is definable from $M_{j}, j \leq \varepsilon_{\ell}$ and $p$, we have $\eta_{\ell} \in M_{\varepsilon_{\ell+1}^{\prime}}$ and hence we can do the preliminary step in $M_{\varepsilon_{\ell+1}^{\prime}}$.

Since $\left.S_{\ell}=h_{*}^{-1}[\{\varrho(\ell))\}\right]$ is in $M_{\varepsilon_{\ell+1}^{\prime}}$ a stationary subset of $\omega_{1}$ there are arbitrary large members of $S_{\ell} \cap\left(M_{\varepsilon_{\ell+1}^{\prime}} \cap \omega_{1}\right)$ and in particular there are members in $S_{\ell} \cap$ $\left(M_{\varepsilon_{\ell+1}^{\prime}} \cap \omega_{1}\right) \cap\left[\alpha_{\delta, n_{\ell+1}}, \alpha_{\delta, n_{\ell+2}}\right)$. Thus there is $\alpha \in \omega_{1} \cap\left[\alpha_{\delta, n_{\ell+1}}, \alpha_{\delta, n_{\ell+2}}\right)$ is such that $h_{*}\left(\eta_{\ell+1}\left(i_{\ell}\right)\right)=\varrho(\ell)$ and $\eta_{\ell}\langle\alpha\rangle \in p$.

Then we choose $\varepsilon_{\ell+1}$ such that $M_{\varepsilon_{\ell+1}} \cap \omega \in\left[\alpha_{\delta, n_{\ell+1}}, \alpha_{\delta, n_{\ell+1}+1}\right)$ and such that $\eta_{\ell+1} \triangleright \eta_{\ell}\langle\alpha\rangle$ is a splitting node of $p$ that has range $\subseteq \alpha_{\delta, n_{\ell+1}+1}$ and $\lg \left(\eta_{\ell+1}\right)=$ $M_{\varepsilon_{\ell+1}} \cap \omega_{1}$.

Why is there such an $\eta_{\ell+1}$ ? Since limits of splitting nodes are splitting nodes and since the sequence $M_{\alpha}$ is continuously increasing, we can find $\eta_{\ell+1}$ by an intermediate $\omega$-sequence of nodes $\eta_{\ell+1, k} \in \operatorname{spl}(p)$ with $M_{\eta_{\ell+1, k}} \cap \omega_{1}=\lg \left(\eta_{\ell+1, k}\right)>M_{\eta_{\ell, k}} \cap \kappa$ and the range of $\eta_{\ell+1, k} \uparrow\left[i_{\ell}, \operatorname{dom}\left(\eta_{\ell+1}\right)\right)$ is a subset of $\left[\alpha_{\delta, n_{\ell+1}}, \alpha_{\delta, n_{\ell+1}+1}\right)$.

By Equations (4.3) and (4.4) the argument $i_{\ell}$ is determined from $\left(n_{\ell}, i_{\ell}\right) \in{\underset{\sim}{w}}_{\delta}$ for some $m \in \mathbb{Z}$, for all $\ell$ and $\left(n_{\ell}+m, h_{*}\left(\underline{\eta}\left(i_{\ell}\right)\right)\right)=\left(n_{\ell}, \varrho(\ell)\right) \in \underline{y}_{\delta} \cdot{ }^{3}$.

In the end we let $\eta=\bigcup \eta_{\ell}$. Now the $n_{\ell}, i_{\ell}, \eta_{\ell+1}, \ell \geq 1$, witness

$$
p^{[\eta]} \Vdash \underset{\sim}{v_{\delta}} E \varrho .
$$

Now we sum up all the cases of $\kappa^{<\kappa}>\kappa$ for regular $\kappa$.

THEOREM 4.3. If $\kappa$ is a regular uncountable cardinal and $\kappa^{<\kappa}>\kappa$ then $\mathbb{Q}_{\kappa}^{a}$ collapses $\kappa^{<\kappa}$ to $\kappa$.

Proof. (1) $\kappa$ is a limit cardinal. Then we can write $\kappa^{<\kappa}$ as $\sup \left\{\kappa^{\theta^{+}}: \theta<\kappa\right\}$. According to Theorem 4.2 part (1) each of the $\kappa^{\theta^{+}}$is collapsed to $\kappa$, and we can combine the names of the collapses and get: Forcing with $\mathbb{Q}_{\kappa}^{\mathrm{a}}$ collapses $\kappa^{<\kappa}$ to $\kappa$.

(2) $\kappa=\theta^{+}$and $\theta>\omega$ is regular. This is Theorem 4.2 part (1).

\footnotetext{
${ }^{3}$ The shift by $m$ may happen since we do not know what $\eta \uparrow \lg \left(\eta_{0}\right)$ determines under the interpretation through Equations (4.3) and (4.4). It might be more or less than what $\varrho$ determines.
} 
(3) $\kappa=\omega_{1}$. This is Theorem 4.2 part (2).

(4) $\kappa=\theta^{+}$and $\operatorname{cf}(\theta)<\theta$.

4.1: The first subcase is: $\kappa^{\theta}=\kappa^{\mu}$ for some $\mu \in\left[\omega_{1}, \theta\right)$. Then we can choose a regular such $\mu$. Now $\kappa^{\theta}=\kappa^{<\kappa}=\kappa^{\mu}$ is collapsed to $\kappa$ by Theorem 4.2 part (1).

4.2: The second subcase is: For all $\mu<\theta, \kappa^{\mu} \leq \kappa$. Then $\kappa^{\theta}=\kappa^{\operatorname{cf}(\theta)}$. This subcase is divided in two subsubcases:

4.2.1: If $\operatorname{cf}(\theta)>\omega$, then by Theorem 4.2 part (1) $\kappa^{\theta}$ is collapsed to $\kappa$.

4.2.2: If $\operatorname{cf}(\theta)=\omega$, then we need to rework part (2) of Theorem 4.2 to get that also $\kappa^{\omega}$ is collapsed. We replace $\omega_{1}$ by $\kappa$ and instead of with countable elementary submodels we work with submodels of cardinality $<\kappa$.

§5. On the Laver property. In this section we assume that $\kappa$ is a measurable cardinal that is measurable after forcing with any $(<\kappa)$-directed forcing. Such a cardinal is gotten by starting with a supercompact cardinal and performing a Laver preparation [15]. Then the supercompactness is indestructible under $(<\kappa)$-directed forcings.

The purpose of this section is to answer a question posed in [4] about the Laver property of an ultrafilter version of Miller forcing. The starting point is the observation in [4] that for normal ultrafilters, each iterand has a version of the Laver property.

Definition 5.1. Given a normal ultrafilter over $\kappa$, we define $\mathbb{Q}_{\kappa}^{\mathrm{a}}(\mathcal{U})$, Miller forcing with branching into $\mathcal{U}$, as follows: A condition $p \in \mathbb{Q}_{\kappa}^{\mathrm{a}}(\mathcal{U})$ is a $(<\kappa)$-closed superperfect tree $p \subseteq{ }^{\kappa>} \kappa$ such that for any splitting node $s \in \operatorname{spl}(p)$,

$$
\operatorname{osucc}_{p}(s)=\left\{\alpha \in \kappa: s^{\wedge}\langle\alpha\rangle \in p\right\}
$$

is an element of $\mathcal{U}$. Subtrees are stronger conditions.

Now we recall the definition of the higher Laver properties.

Definition 5.2 (See [4, Definition 79]). Let $h: \kappa \rightarrow \kappa \backslash\{0\}$. A sequence $\left\langle\varphi_{\alpha}\right.$ : $\alpha\langle\kappa\rangle$ is called a $h$-slalom if for each $\alpha, \varphi_{\alpha} \in[\kappa]^{|h(\alpha)|}$. We say $\mathbb{P}$ has the $h$-Laver property if for any $\mathbb{P}$ name $\tau$ and for any $g: \kappa \rightarrow \kappa$ such that $\mathbb{P} \Vdash \tau{ }_{\sim} \leq^{*} g$ there is a $h$-slalom $\left\langle\varphi_{\alpha}: \alpha<\kappa\right\rangle$ and there is $q$ such that

$$
q \Vdash\left|\left\{\alpha<\kappa: \underset{\sim}{\tau}(\alpha) \notin \varphi_{\alpha}\right\}\right|<\kappa .
$$

We answer a question by Brendle, Brooke-Taylor, Friedman, and Montoya:

Proposition 5.3. Assume that $\kappa$ is supercompact and underwent the Laver preparation. Let $\mathbb{P}=\left\langle\mathbb{P}_{m}, \mathbb{Q}_{n}: n<\omega, m \leq \omega\right\rangle$ be the countable support iteration of length $\omega$ of iterands $\mathbb{Q}_{n}=\mathbb{Q}_{\kappa}^{a}\left(\mathcal{U}_{n}\right)$, where $\mathcal{U}_{n}$ is a normal ultrafilter over $\kappa$ in $\mathbf{V}^{\mathbb{P}_{n}}$. By the Laver preparation $\kappa$ is still supercompact in $\mathbf{V}^{\mathbb{P}_{n}}$. For any $h: \kappa \rightarrow \kappa \backslash\{0\}$ there is a $g: \kappa \rightarrow \kappa$ and there is a $\mathbb{P}$-name for a function below $g$ that witnesses that $\mathbb{P}$ does not have the h-Laver property.

Proof. Let $h$ be given. For each $\delta<\kappa$ we choose some cardinal $g(\delta) \in$ $\left[|h(\delta)|^{+}, \kappa\right)$. For any $\delta<\kappa$ fix a partition $\left\langle S_{\delta, \varepsilon}: \varepsilon<g(\delta)\right\rangle$ of $S_{\aleph_{0}}^{\kappa}=\{\alpha<\kappa$ : $\left.\operatorname{cf}(\alpha)=\aleph_{0}\right\}$ into $g(\delta)$ many stationary sets. 
Let $\eta_{n}$ be the $\mathbb{Q}_{n}$-generic $\kappa$-real.

Now we define a name for a function that is bounded by $g$ :

$$
v(\delta)= \begin{cases}\varepsilon, & \text { if } \lim _{n}\left\langle\eta_{n}(\delta): n<\omega\right\rangle \in S_{\delta, \varepsilon} \\ 0, & \text { else. }\end{cases}
$$

Now we show:

$(*)\left(\forall \varrho \in \prod_{\delta<\kappa} g(\delta)\right)^{\mathbf{V}}$, for any condition $p$ for any $\varrho$-slalom $\bar{T}=\langle T(\delta): \delta<\kappa\rangle$ in the ground model, for any club $C$ of $\delta<\kappa$, there is some $\delta \in C, p \Vdash v(\delta) \notin$ $T(\delta))$.

Property $(*)$ can be applied in particular to $\varrho=h, C=[\alpha, \kappa)$ for some $\alpha<\kappa$, and hence negates the $h$-Laver property. Let $\left.\varrho \in \prod_{\delta<\kappa} g(\delta)\right)^{\mathbf{V}},\langle T(\delta): \delta<\kappa\rangle \in \mathbf{V}$ be such that $T(\delta) \in[\kappa]^{\varrho(\delta)}$ for $\delta<\kappa, p$ be a condition and let $\underset{\sim}{C}$ be a $\mathbb{P}$-name for a club subset of $\kappa$. We show that

$(\odot)$ There are $q \geq p$ and $\delta<\kappa$ such that $q \Vdash \delta \in \underset{\sim}{C \wedge} \wedge(\delta) \notin T(\delta)$.

By induction on $n<\omega$ we choose $p_{n}, \delta_{n}$ such that $\tilde{p_{n}}=\left\langle p_{n}(k): k \in \omega\right\rangle$, where $k$ is the coordinate that runs over the iteration stages, such that

(1) $p_{0}=p, \delta_{0}=0$,

(2) $p_{n+1} \geq p_{n}, \operatorname{dom}\left(p_{n}\right)=\omega$,

(3) $p_{n} \uparrow k \Vdash \operatorname{tr}\left(p_{n}(k)\right)=\eta_{n, k}$,

(4) $\delta_{n+1}>\delta_{n}$,

(5) $p_{n} \uparrow k \Vdash \delta_{n+1}>\lg \left(\eta_{n, k}\right)$,

(6) $p_{n+1} \Vdash \delta_{n+1} \in C$, and

(7) $p_{n+1} \uparrow k \Vdash_{\mathbb{P}_{k}} \lg \left(\operatorname{tr}\left(p_{n+1}(k)\right) \geq \delta_{n+1}\right.$.

In the end we use that $\mathbb{P}_{\omega}$ is $(<\kappa)$-closed and hence we can let $p_{\omega}$ be a lower bound of $p_{n}, n<\omega$. We have

$$
p_{\omega} \Vdash \delta \in \underset{\sim}{C} .
$$

Since $|T(\delta)|<g(\delta)$, we can choose an $\varepsilon \in g(\delta) \backslash T(\delta)$. Then we choose $N_{n} \prec$ $(H(\chi), \in)$ such that

(1) $\bar{T}, \mathbb{P}, p_{\omega} \in N_{0}$

(2) $\left|N_{n}\right|<\kappa$

(3) $N_{n} \cap \kappa \in S_{\delta, \varepsilon}$, and

(4) $N_{n} \in N_{n+1}$.

So there is $q_{1} \geq p_{\omega}$ such that $q_{1} \in N_{1} \cap \mathbb{P}, q_{1}(0) \geq p_{\omega}(0), q_{1}(n)=p_{\omega}(n)$ for $n>0$. By induction on $n$ we choose $q_{n}$ such that

$$
q_{n} \uparrow(n+1) \Vdash \eta_{n}(\delta) \in\left[N_{n} \cap \kappa, N_{n+1} \cap \kappa\right)
$$

and $q_{n}(k)=p_{\omega}(k)$ for $k>n$. Let $q_{\omega}$ be an upper bound of the $q_{n}$. Then

$$
q_{\omega} \Vdash \lim _{n} \eta_{n}(\delta)=\lim _{n}\left(N_{n} \cap \kappa\right) \in S_{\delta, \varepsilon} .
$$

Hence by Equation (5.1),

$$
q_{\omega} \Vdash v(\delta)=\varepsilon \in \kappa \backslash T(\delta),
$$

and $\odot$ is proved. 
Acknowledgments. This research, no. 1191 (Saharon Shelah), was partially supported by European Research Council grant 338821. The authors thank the Technical University of Vienna and the Institute of Discrete Mathematics and Geometry for their hospitality.

\section{REFERENCES}

[1] B. BALCAR and P. SimON, Disjoint refinement, Handbook of Boolean Algebras, vol. 2 (J. Donald Monk and R. Bonnet, editors), North-Holland, Amsterdam, 1989, pp. 333-388.

[2] J. Baumgartner, Almost-disjoint sets, the dense-set problem, and the partition calculus. Annals of Mathematical Logic, vol. 9 (1976), pp. 401-439.

[3] J. Baumgartner and R. LAVER, Iterated perfect-set forcing. Annals of Mathematical Logic, vol. 17 (1979), pp. 271-288.

[4] J. Brendle, A. Brooke-Taylor, S.-D. Friedman, and D. C. Montoya, Cichoń's diagram for uncountable cardinals. Israel Journal of Mathematics, vol. 225 (2018), no. 2, pp. 959-1010.

[5] J. Cummings and S. Shelah, Cardinal invariants above the continuum. Annals of Pure and Applied Logic, vol. 75 (1995), pp. 251-268.

[6] T. EIswORTH, On iterated forcing for successors of regular cardinals. Fundamenta Mathematicae, vol. 179 (2003), no. 3, pp. 249-266.

[7] S.-D. Friedman and L. ZdomsKyY, Measurable cardinals and the cofinality of the symmetric group. Fundamenta Mathematicae, vol. 207 (2010), no. 2, pp. 101-122.

[8] M. Golshani, (Weak) diamond can fail at the least inaccessible cardinal, preprint 2016, https://arxiv.org/abs/1607.00751.

[9] T. JeCH, Set Theory . The Third Millenium Edition, revised and expanded. Springer, Berlin, 2003.

[10] A. Kanamori, Perfect-set forcing for uncountable cardinals. Annals of Mathematical Logic, vol. 19 (1980), nos. 1-2, pp. 97-114.

[11] M. KoJman and S. SHELAH, Non-existence of universal orders in many cardinals, this Journal, vol. 57 (1992), pp. 875-891.

[12] K. Kunen, Set Theory, An Introduction to Independence Proofs. North-Holland, Amsterdam, 1980 .

[13] G. LaguzZI, Uncountable trees and Cohen reals, preprint, 2019. http://home.mathematik. uni-freiburg.de/giorgio/publist.html.

[14] A. LANDVER, Baire numbers, uncountable Cohen sets and perfect-set forcing. this JournaL, vol. 57 (1992), no. 3, pp. 1086-1107.

[15] R. LAVER, Making the supercompactness of $\kappa$ indestructible under $\kappa$-directed closed forcing. Israel Journal of Mathematics, vol. 29 (1978), pp. 385-388.

[16] A. Miller, Rational perfect set forcing, Axiomatic Set Theory (J. Baumgartner, D. A. Martin, and S. Shelah, editors), Contemporary Mathematics, 31, American Mathematical Society, Providence, 1984, pp. 143-159.

[17] A. Roslanowski and S. Shelah, Iteration of $\lambda$-complete forcing notions not collapsing $\lambda^{+}$. International Journal of Mathematics and Mathematical Sciences, vol. 28 (2001), pp. 63-82.

[18] —, Sheva-Sheva-Sheva: large creatures. Israel Journal of Mathematics, vol. 159 (2007), pp. $109-174$.

[19] - More about $\lambda$-support iterations of $(<\lambda)$-complete forcing notions. Archive for Mathematical Logic, vol. 52 (2013), pp. 603-629.

[20] - The last forcing standing with diamonds. Fundamenta Mathematicae, vol. 246 (2019), no. 2, pp. 109-159.

[21] S. Shelah, Models with second order properties. IV. A general method and eliminating diamonds. Annals of Pure and Applied Logic, vol. 25 (1983), pp. 183-212.

[22] - Reflecting stationary sets and successors of singular cardinals. Archive for Mathematical Logic, vol. 31 (1991), pp. 25-53.

[23] - Proper and Improper Forcing, Perspectives in Mathematical Logic, Springer, Berlin, 1998.

[24] - Applications of PCF theory, this Journal, vol. 65 (2000), pp. 1624-1674.

[25] - More on the revised GCH and the black box. Annals of Pure and Applied Logic, vol. 140 (2006), pp. 133-160. 
[26] - Power set modulo small, the singular of uncountable cofinality, this JOURnAL, vol. 72 (2007), pp. 226-242.

[27] - Diamonds. Proceedings of the American Mathematical Society, vol. 138 (2010), pp. 2151-2161.

[28] P. SimON, Sacks forcing collapsesctob. Commentationes Mathematicae Universitatis Carolinae, vol. 34 (1993), no. 4, pp. 707-710.

[29] M. Zeman, $\diamond$ at Mahlo cardinals, this JournaL, vol. 65 (2000), no. 4, pp. 1813-1822.

\author{
ALBERT-LUDWIGS-UNIVERSITÄT FREIBURG \\ MATHEMATISCHES INSTITUT, ABTEILUNG FÜR MATH. LOGIK \\ ERNST-ZERMELO-STRASSE 1 \\ 79104 FREIBURG IM BREISGAU, GERMANY \\ E-mail: heike.mildenberger@math.uni-freiburg.de \\ INSTITUTE OF MATHEMATICS \\ THE HEBREW UNIVERSITY OF JERUSALEM \\ EDMOND SAFRA CAMPUS GIVAT RAM \\ 9190401, JERUSALEM, ISRAEL \\ E-mail:shelah@math.huji.ac.il
}

